\title{
THE FREDHOLM METHOD IN POTENTIAL THEORY $\left({ }^{1}\right)$
}

\author{
BY \\ JOSEF KRÁL
}

Introduction. Let $G$ be an open set with a compact boundary $B$ in $R^{m}$, the Euclidean $m$-space. If $h$ is a harmonic function in $G$ such that

$$
\int_{P}|\operatorname{grad} h(x)| d x<\infty
$$

for every bounded open set $P \subset G$, one may form the distribution $N h$ over the space $D$ of all infinitely differentiable functions $\psi$ with compact support in $R^{m}$ defining

$$
\langle\psi, N h\rangle=\int_{G} \operatorname{grad} \psi(x) \cdot \operatorname{grad} h(x) d x .
$$

This distribution will be termed the generalized normal derivative of $h$ (compare [CC], [M], [Y]). It is easily seen that $N h$ has support in $B$. In general, $N h$ need not be a measure in the sense usual in distribution theory [S]. $\S 1$ of the present paper deals with generalized normal derivatives of Newtonian potentials. We denote by $C^{*}(B)$ the Banach space of all finite signed Borel measures with support in $B$; total variation is taken as a norm in $C^{*}(B)$. With every $\mu \in C^{*}(B)$ we associate the corresponding Newtonian potential

$$
U \mu(x)=\int_{R^{m}} p(x-y) d \mu(y),
$$

where $p(z)=|z|^{2-m} / m-2$ or $p(z)=\log (1 /|z|)$ according as $m>2$ or $m=2$, and we ask what necessary and sufficient condition is to be imposed on $G$ in order that $N U \mu$ be a measure for every $\mu \in C^{*}(B)$. For this purpose it is useful to introduce the concept of a hit of a half-line $\{y+t \theta: t>0\}$ on $G$ (cf. Definition 1.5). If $n(\theta, y)$ denotes the number of such hits, then $n(\theta, y)$ is a Baire function of the variable $\theta$ on $\Gamma=R^{m} \cap\{\theta:|\theta|=1\}$ and the above mentioned condition reads as follows:

$$
\sup _{y \in B} \int_{\Gamma} n(\theta, y) d H_{m-1}(\theta)<\infty,
$$

where $H_{m-1}$ stands for the $(m-1)$-dimensional Hausdorff measure.

Presented to the Society, January 27, 1966 under the title Normal derivatives of potentials, received by the editors May 31, 1966.

( ${ }^{1}$ This work was supported by the National Science Foundation, United States of America. 
If $G$ fulfills (2), then the operator

$$
N U: \mu \rightarrow N U \mu
$$

is bounded on $C^{*}(B)$ and has the form $\frac{1}{2} A I+\bar{W}^{*}$, where $A=H_{m-1}(\Gamma), I$ is the identity operator and $\bar{W}^{*}$ is adjoint to an operator $\bar{W}$ acting on the space $C(B)$ of all continuous functions on $B$. Some properties of $\bar{W}$, which is connected with the classical double-layer potential, are investigated in $\$ \$ 2-3$. In particular, in $\S 3$ we show that, in case $B$ has no isolated points, the Fredholm radius of $\bar{W}$ is the reciprocal of the quantity

$$
V_{0}=\lim _{r \downarrow 0} \sup _{y \in B}\left[A\left|d(y)-\frac{1}{2}\right|+\int_{\Gamma} n_{r}(\theta, y) d H_{m-1}(\theta)\right],
$$

where $d(y)$ denotes the $m$-density of $G$ at $y$ and $n_{r}(\theta, y)$ is the number of hits of $\{y+t \theta: 0<t<r\}$ on $G$. Relations between $V_{0}$ and the geometric structure of $B$ are also investigated in $\S 3$. In case $V_{0}$ is sufficiently small, these results apply to the Neumann problem where the boundary condition is given by an arbitrary measure $\nu \in C^{*}(B)$, as treated in $\$ 4$. By duality based on the Fredholm theory one obtains, as a by-product, representation of solutions of the Dirichlet problem by means of double-layer potentials.

Methods and concepts employed here are those of geometric measure theory; they have their origin in investigations connected with the Gauss-Green theorem, sets with finite perimeter and functions whose partial derivatives are measures [DG], [F], [FL], [FY], [KR], [MA], [P].

\section{Normal derivatives of potentials.}

1.1. Terminology and notation. The symbols $R^{m}, C^{*}(B), p, U_{\mu}, D$ will have the meaning described in the introduction. For $M \subset R^{m}$ we shall denote by $\mathrm{cl} M$, int $M$, fr $M$ and diam $M$ the closure, interior, boundary and diameter of $M$, respectively. $H_{k}$ will stand for the $k$-dimensional Hausdorff measure; $H_{m}$ coincides with the Lebesgue measure in $R^{m}$. We put $\Omega_{r}(y)=R^{m} \cap\{z:|z-y|<r\}, \quad \Omega=\Omega_{1}(0)$, $\Gamma_{r}(y)=$ fr $\Omega_{r}(y), \Gamma=\Gamma_{1}(0), A=H_{m-1}(\Gamma)$. Throughout this paragraph $G \subset R^{m}(m \geqq 2)$ will be a fixed set with a compact boundary $B$. We shall tacitly assume that $G$ is open. On several places, however, it will be useful to allow $G$ to be a Borel set; this will be always pointed out explicitly.

The generalized normal derivative of a harmonic function $h$ (satisfying (1) for every bounded open $P \subset G$ ) is defined as in the introduction; we shall write $N^{G} h$ instead of $N h$ if it is necessary to specify $G$. The reason for the terminology is obvious: if $G$ has a smooth boundary with exterior normal $n$ and $h$ is smooth up to $B$, then

$$
\langle\psi, N h\rangle=\int_{B} \psi(\partial h / \partial n) d H_{m-1}
$$


If $\operatorname{spt} \psi$ (= the support of $\psi$ ) does not meet $B$, then there is an open set $Q$ with a smooth boundary such that spt $\psi \cap G \subset Q, \mathrm{cl} Q \subset G$, so that

$$
\left\langle\psi, N^{G} h\right\rangle=\left\langle\psi, N^{Q} h\right\rangle=0 .
$$

In particular, if $N^{G} h$ is a (Borel) measure $\nu$, which means that

$$
\left\langle\psi, N^{a} h\right\rangle=\int_{R^{m}} \psi d \nu
$$

for every $\psi \in D$, then $\nu \in C^{*}(B)$.

Variation of a (signed) measure $\mu$ on a Borel set $M$ will be denoted by $|\mu|(M)$; for $\mu \in C^{*}(B),|\mu|(B)=\|\mu\|$ is the norm of $\mu$.

Simple calculation shows that, for $\mu \in C^{*}(B)$ and $x \in G$,

$$
|\operatorname{grad} U \mu(x)| \leqq \int_{B}|x-y|^{1-m} d|\mu|(y),
$$

whence we obtain for any bounded Borel $P \subset G$

$$
\int_{P}|\operatorname{grad} U \mu(x)| d x \leqq A \operatorname{diam}(B \cup P)\|\mu\| .
$$

We see that $N U \mu$ is meaningful for every $\mu \in C^{*}(B)$. Our main objective in $\S 1$ is to answer the following question:

1.2. What necessary and sufficient restrictions are to be imposed on $G$ in order that $N U_{\mu}$ be a measure for every $\mu \in C^{*}(B)$ ?

1.3. REMARK. Let us agree to denote by $\delta_{y}$ the Dirac measure concentrated at $y \in R^{m}$. We have for any $\psi \in D$ and any $y \in B$

$$
\left\langle\psi, N U \delta_{y}\right\rangle=\int_{G} \operatorname{grad} \psi(x) \cdot \frac{y-x}{|y-x|^{m}} d x .
$$

Direct calculation shows that, in case $Q=R^{m}-\{y\}, N^{Q} U \delta_{y}=A \delta_{y}$.

Let us also observe that, for $\psi \in D$ and $\mu \in C^{*}(B)$,

$$
\langle\psi, N U \mu\rangle=\int_{B}\left\langle\psi, N U \delta_{y}\right\rangle d \mu(y) .
$$

Indeed, if $P=G \cap \operatorname{spt} \psi$ and $K=\sup |\operatorname{grad} \psi|$, then

$$
\iint_{G \times B}\left|\operatorname{grad} \psi(x) \cdot \frac{y-x}{|y-x|^{m}}\right| d x d|\mu|(y) \leqq K A \operatorname{diam}(P \cup B)\|\mu\|,
$$

so that Fubini's theorem applies to

$$
\iint_{G \times B} \operatorname{grad} \psi(x) \cdot(y-x)|y-x|^{-m} d x d \mu(y) ;
$$

it remains to notice that the two repeated integrals derived from (1.3) occur in (1.2). 
Before investigating the problem 1.2 we shall answer the following simpler question:

1.4. Fix $y \in B$. What must be the shape of $G$ in order that $N U \delta_{y}$ be a measure?

Let us first introduct a concept which will be useful later.

1.5. Definition. If $M \subset R^{k}$ is a Borel set and $S \subset R^{k}$ is an open segment or halfline then $z \in S$ will be termed a hit of $S$ on $M$ provided both $S \cap M \cap \Omega_{r}(z)$ and $(S-M) \cap \Omega_{r}(z)$ have a positive linear measure for every $r>0$.

An answer to 1.4 is included in the following proposition, which will be needed later.

1.6. Proposition. Suppose that $G$ is a Borel set. Fix $y \in R^{m}, r>0$ and put

$$
\begin{aligned}
& E_{r}(y)=D \cap\left\{\psi: \operatorname{spt} \psi \subset \Omega_{r}(y),|\psi| \leqq 1\right\}, \\
& D_{r}(y)=E_{r}(y) \cap\{\psi: y \notin \operatorname{spt} \psi\} .
\end{aligned}
$$

If $n_{r}(\theta, y)$ denotes the number (possibly 0 or $\infty$ ) of all hits of $\{y+\rho \theta: 0<\rho<r\}$ on $G$, then $n_{r}(\theta, y)$ is a Baire function of the variable $\theta$ on $\Gamma$, the integral

$$
v_{r}(y)=\int_{\Gamma} n_{r}(\theta, y) d H_{m-1}(\theta)
$$

is equal to

$$
\sup \left\{\int_{G} \operatorname{grad} \psi(x) \cdot \frac{y-x}{|y-x|^{m}} d x: \psi \in D_{r}(y)\right\}
$$

and

$$
\sup \left\{\int_{G} \operatorname{grad} \psi(x) \cdot \frac{y-x}{|y-x|^{m}} d x: \psi \in E_{r}(y)\right\} \leqq A+v_{r}(y) .
$$

If $y \in B$ and $G$ is open, then $N U \delta_{y}$ is a measure if and only if $v_{\infty}(y)<\infty$.

1.7. REMARK. If it is necessary to specify the set $G$, we write $n_{r}^{G}(\theta, y)$ and $v_{r}^{G}(y)$ instead of $n_{r}(\theta, y)$ and $v_{r}(y)$.

We postpone the proof of Proposition 1.6 to 1.11. First we establish two lemmas.

1.8. Notation. If $f$ is a function in $R^{1}$ we denote by var $[f ;(a, b)]$ its variation on $(a, b)=R^{1} \cap\{t: a<t<b\}$. If $f$ is known to be summable over every compact subset in $(a, b)$, we shall use var ess $[f ;(a, b)]$ to denote $\sup _{\psi} \int_{a}^{b} \psi^{\prime}(t) f(t) d t, \psi$ ranging over all infinitely differentiable functions with compact spt $\psi \subset(a, b)$ such that $|\psi| \leqq 1$.

REMARK. It follows easily from the Riesz representation theorem and elementary distribution theory that var ess $[f ;(a, b)]<\infty$ implies the existence of a function $g$ in $(a, b)$ such that $g=f$ a.e. in $(a, b)$ and $\operatorname{var}[g ;(a, b)]=\operatorname{var}$ ess $[f ;(a, b)]$.

Clearly, $\operatorname{var}[f ;(a, b)]=\operatorname{var}$ ess $[f ;(a, b)]$ whenever $f$ is continuous in $(a, b)$.

1.9. LemMA. If $c_{M}$ is the characteristic function of a Borel set $M \subset R^{1}$, then var ess $\left[c_{M} ;(a, b)\right]$ equals the number of hits of $(a, b)$ on $M$. 
Proof. Let $q$ stand for the number of all hits of $(a, b)$ on $M$. If $q<\infty$ and $a_{1}<\cdots<a_{q}$ are all the hits, then no $\left(a_{j}, a_{j+1}\right)$ can meet both $M$ and $(a, b)-M$ in a set of positive linear measure. It follows that either $M$ or $(a, b)-M$ is equivalent with $\bigcup_{k}\left(a_{2 k-1}, a_{2 k}\right)$, where $1 \leqq k, 2 k \leqq q$. Consequently, var ess $\left[c_{M} ;(a, b)\right]=q$. Conversely, if var ess $\left[c_{M} ;(a, b)\right]<\infty$, then there is a $g$ with var $[g ;(a, b)]<\infty$ such that $g=c_{M}$ a.e. in $(a, b)$.

Clearly, this implies $q<\infty$.

1.10. Lemma. Let $f$ be a bounded Baire function in $R^{m}, y \in R^{m}, 0 \leqq a<b \leqq \infty$. For $\theta \in \Gamma$ put

$$
f_{\theta}(t)=f(y+t \theta), \quad t \in R^{1} .
$$

Then var ess $\left[f_{\theta} ;(a, b)\right]$ is a Baire function of the variable $\theta$ on $\Gamma$ and the integral

$$
\int_{\Gamma} \operatorname{var} \operatorname{ess}\left[f_{\theta} ;(a, b)\right] d H_{m-1}(\theta)
$$

equals

$$
v(a, b, f)=\sup _{\psi} \int_{R^{m}} f(x) \operatorname{grad} \psi(x) \cdot \frac{y-x}{|y-x|^{m}} d x,
$$

$\psi$ ranging over all functions in $D$ with

$$
\operatorname{spt} \psi \subset R^{m} \cap\{x: a<|x-y|<b\}, \quad|\psi| \leqq 1 .
$$

Proof. We may assume $y=0, b<\infty$. Using the notation from (1.4) we obtain for any $\psi \in D$ satisfying (1.5)

$$
\begin{aligned}
\int_{R^{m}} f(x) \operatorname{grad} \psi(x) \cdot \frac{x}{|x|^{m}} d x & =\int_{\Gamma}\left(\int_{a}^{b} f_{\theta}(t) \psi_{\theta}^{\prime}(t) d t\right) d H_{m-1}(\theta), \\
\int_{a}^{b} f_{\theta}(t) \psi_{\theta}^{\prime}(t) d t & \leqq \operatorname{var} \operatorname{ess}\left[f_{\theta} ;(a, b)\right] .
\end{aligned}
$$

Assuming that we know already that var ess $\left[f_{\theta} ;(a, b)\right]$ is measurable $\left(H_{m-1}\right)$ on $\Gamma$ we get

$$
v(a, b, f) \leqq \int_{\Gamma} \operatorname{var} \text { ess }\left[f_{\theta} ;(a, b)\right] d H_{m-1}(\theta) .
$$

It remains to prove that var ess $\left[f_{\theta} ;(a, b)\right]$ is a Baire function of $\theta$ and

$$
\int_{\Gamma} \operatorname{var} \operatorname{ess}\left[f_{\theta} ;(a, b)\right] d H_{m-1}(\theta) \leqq v(a, b, f) .
$$

To show this we first assume, in addition, that

(I). $f_{\theta}$ has a continuous derivative on $(a, b)$ for every $\theta \in \Gamma$ and

$$
\sup \left\{\left|f_{\theta}^{\prime}(t)\right|: \theta \in \Gamma, c<t<d\right\}=K(c, d)<\infty
$$

whenever $a<c<d<b$. 
For every positive integer $N$ we subdivide $(a, b)$ by means of points

$$
a_{k}=a_{k}^{N}=a+k 2^{-N}(b-a), \quad 1 \leqq k<2^{N} .
$$

Consider $k<2^{N}-2$. Since sign $\left[f_{\theta}\left(a_{k+1}\right)-f_{\theta}\left(a_{k}\right)\right]$ is a Baire function of $\theta$, there are functions $\phi_{k s} \in D$ such that $\left|\phi_{k s}\right| \leqq 1$ and

$$
\lim _{s \rightarrow \infty} \phi_{k s}(\theta)=\operatorname{sign}\left[f_{\theta}\left(a_{k+1}\right)-f_{\theta}\left(a_{k}\right)\right] \quad \text { a.e. }\left(H_{m-1}\right)
$$

on $\Gamma$. Further express the characteristic function of $\left(a_{k}, a_{k+1}\right)$ as $\lim _{s \rightarrow \infty} \rho_{k s}$, where $\rho_{k s}$ are infinitely differentiable functions in $R^{1}$ with

$$
\text { spt } \rho_{k s} \subset\left(a_{k}, a_{k+1}\right),\left|\rho_{k s}\right| \leqq 1,
$$

and define

$$
\psi_{s}(t \theta)=-\sum_{k=1}^{2^{N}-2} \phi_{k s}(\theta) \rho_{k s}(t), \quad t \geqq 0, \theta \in \Gamma .
$$

Then

$$
\psi_{s} \in D,\left|\psi_{s}\right| \leqq 1, \text { spt } \psi_{s} \subset R^{m} \cap\{x: a<|x|<b\} .
$$

Consequently,

$$
v(a, b, f) \geqq \int_{\Gamma}\left[\int_{a}^{b} f_{\theta}(t) \psi_{s \theta}^{\prime}(t) d t\right] d H_{m-1}(\theta) .
$$

The sequence of integrals

$$
\int_{a}^{b} f_{\theta}(t) \psi_{s \theta}^{\prime}(t) d t=\sum_{k=1}^{2^{N}-2} \phi_{k s}(\theta) \int_{a_{k}}^{a_{k+1}} \rho_{k s}(t) f_{\theta}^{\prime}(t) d t
$$

is dominated by $(b-a) K\left(a_{1}, a_{2^{N}-1}\right)$ and converges, as $s \rightarrow \infty$, to

$$
\sigma_{N}(\theta)=\sum_{k=1}^{2^{N}-2}\left|f_{\theta}\left(a_{k+1}\right)-f_{\theta}\left(a_{k}\right)\right|
$$

a.e. $\left(H_{m-1}\right)$ on $\Gamma$. Hence we conclude

$$
v(a, b, f) \geqq \int_{\Gamma} \sigma_{N}(\theta) d H_{m-1}(\theta) .
$$

Noting that $\sigma_{N}(\theta) \uparrow \operatorname{var}\left[f_{\theta} ;(a, b)\right]$ as $N \rightarrow \infty$ we see that var $\left[f_{\theta} ;(a, b)\right]$ is a Baire function of $\theta$ and (1.6) holds in this special case.

Let us now drop the additional assumptions (I) on $f$. For every positive integer $N$ we fix a symmetric infinitely differentiable function $\omega_{N}$ in $R^{1}$ with

$$
\text { spt } \omega_{N} \subset(-1 / N, 1 / N), \int_{R^{1}} \omega_{N}(t) d t=1
$$


and define $f_{N}$ so that $f_{N \theta}=f_{\theta} * \omega_{N}$ (=the convolution of $f_{\theta}$ and $\omega_{N}$ ) on the positive real axis, $f_{N}(0)=0$. Let $a^{N}=a+1 / N, b^{N}=b-1 / N, 2 / N<b-a$. It follows from the first part of the proof that

$$
\int_{\Gamma} \operatorname{var}\left[f_{N \theta} ;\left(a^{N}, b^{N}\right)\right] d H_{m-1}(\theta)=v\left(a^{N}, b^{N}, f_{N}\right)
$$

If $\psi_{N}$ is obtained from $\psi$ in the same way as $f_{N}$ from $f$, then

$$
\psi \in D,|\psi| \leqq 1, \text { spt } \psi \subset R^{m} \cap\left\{x: a^{N}<|x|<b^{N}\right\}
$$

imply

$$
\psi_{N} \in D,\left|\psi_{N}\right| \leqq 1, \text { spt } \psi_{N} \subset R^{m} \cap\{x: a<|x|<b\}
$$

and

$$
\int_{a^{N}}^{b^{N}} \psi_{\theta}^{\prime}(t) f_{N \theta}(t) d t=\int_{a}^{b} \psi_{N \theta}^{\prime}(t) f_{\theta}(t) d t
$$

Consequently,

$$
v\left(a^{N}, b^{N}, f_{N}\right) \leqq v(a, b, f) .
$$

The same argument shows that

$$
\operatorname{var} \operatorname{ess}\left[f_{N \theta} ;\left(a^{N}, b^{N}\right)\right] \leqq \operatorname{var} \operatorname{ess}\left[f_{\theta} ;(a, b)\right] .
$$

It is easy to see that

$$
\liminf _{N \rightarrow \infty} \operatorname{var} \operatorname{ess}\left[f_{N \theta} ;\left(a^{N}, b^{N}\right)\right] \geqq \operatorname{var} \operatorname{ess}\left[f_{\theta} ;(a, b)\right] \text {, }
$$

which together with (1.9) yields

$$
\lim _{N \rightarrow \infty} \operatorname{var} \operatorname{ess}\left[f_{N \theta} ;\left(a^{N}, b^{N}\right)\right]=\operatorname{var} \operatorname{ess}\left[f_{\theta} ;(a, b)\right] .
$$

In particular, var ess $\left[f_{\theta} ;(a, b)\right]$ is a Baire function of $\theta$. (1.7), (1.8), and (1.10) imply (1.6).

REMARK. The above lemma could also be derived from general theorems on functions, whose partial derivatives are measures; cf. [FL], [KR], [P] on the subject.

Now it is easy to present the following.

1.11. Proof of Proposition 1.6. Let $f$ be the characteristic function of $G$. By 1.9 and 1.10

$$
\begin{aligned}
\operatorname{var} \text { ess }\left[f_{\theta} ;(0, r)\right] & =n_{r}(\theta, y), \\
v_{r}(y) & =v(0, r, f) .
\end{aligned}
$$

If $n_{r}(\theta, y)<\infty$, then $G \cap\{y+t \theta: 0<t<r\}$ is equivalent $\left(H_{1}\right)$ with a finite union 
of disjoint segments, whose end points are hits of $\{y+t \theta: 0<t<r\}$ on $G$ and, possibly, $y$ and $y+r \theta$. Hence we conclude for $\psi \in E_{r}(y)$

$$
\begin{gathered}
\left|\int_{0}^{\infty} f_{\theta}(t) \psi_{\theta}^{\prime}(t) d t\right| \leqq 1+n_{r}(\theta, y), \quad \theta \in \Gamma, \\
\int_{G} \operatorname{grad} \psi(x) \cdot(y-x)|y-x|^{-m} d x \leqq \int_{\Gamma}\left[1+n_{r}(\theta, y)\right] d H_{m-1}(\theta)=A+v_{r}(y) .
\end{gathered}
$$

It remains to note that, in case $y \in B$ and $G$ is open, $N U \delta_{y}$ is a measure if and only if

$$
\sup \left\{\left\langle\psi, N U \delta_{y}\right\rangle: \psi \in D,|\psi| \leqq 1\right\}<\infty .
$$

1.12. RemarK. Let us observe that, in case $y \in B$ and $N U \delta_{y} \in C^{*}(B)$,

$$
v_{\infty}(y) \leqq\left\|N U \delta_{y}\right\| \leqq A+v_{\infty}(y) .
$$

Now we are in position to answer the question raised in 1.2.

1.13. THEOREM. $N U \mu$ is a measure for every $\mu \in C^{*}(B)$ if and only if

$$
V=\sup _{y \in B} v_{\infty}(y)<\infty \text {. }
$$

If this is the case, then

$$
N U: \mu \rightarrow N U \mu
$$

is a bounded linear operator on $C^{*}(B)$,

$$
\|N U\| \leqq A+V
$$

and (1.2) holds for every bounded Baire function $\psi$ on B. In particular,

$$
N U_{\mu}(M)=\int_{B} N U \delta_{y}(M) d \mu(y)
$$

for $\mu \in C^{*}(B)$ and every Borel set $M \subset B$.

Proof. With every $\psi \in D$ we associate a linear functional $L_{\psi}$ over $C^{*}(B)$ defined by

$$
\left\langle\mu, L_{\psi}\right\rangle=\left\langle\psi, N U_{\mu}\right\rangle, \quad \mu \in C^{*}(B) .
$$

Denoting

$$
P_{\psi}=G \cap \operatorname{spt} \psi, \quad s_{\psi}=\sup |\operatorname{grad} \psi|,
$$

we obtain from (1.1)

$$
\left|\left\langle\mu, L_{\psi}\right\rangle\right| \leqq s_{\psi} A \operatorname{diam}\left(B \cup P_{\psi}\right)\|\mu\|
$$

which shows that every $L_{\psi}$ is bounded on $C^{*}(B)$. Let $E=D \cap\{\psi:|\psi| \leqq 1\}$. Then $N U_{\mu}$ is a measure if and only if

$$
\sup _{\psi \in E}\left\langle\psi, N U_{\mu}\right\rangle<\infty .
$$


In particular, if $N U \mu$ is a measure for every $\mu \in C^{*}(B)$, then the class of functionals $\left\{L_{\psi}\right\}_{\psi \in E}$ must be pointwise bounded on $C^{*}(B)$ and, by the uniform boundedness principle,

$$
\sup _{\forall \in E}\left\|L_{\psi}\right\|=K<\infty \text {. }
$$

Employing (1.11) we get for every $y \in B$

$$
v_{\infty}(y) \leqq \sup _{v \in E}\left\langle\psi, N U \delta_{y}\right\rangle \leqq K .
$$

Conversely, if (1.12) holds, then (1.2) together with (1.11) imply

$$
\sup _{\nu \in E}|\langle\psi, N U \mu\rangle| \leqq(A+V)\|\mu\|
$$

for every $\mu \in C^{*}(B)$. It is also easily seen that in this case (1.2) extends to any bounded Baire function $\psi$.

2. Double layer potentials.

2.1. Notation. Throughout this paragraph $C \subset R^{m}$ will denote a Borel set with a compact boundary $B$. Given $z \in R^{m}$ we put

$$
D(z)=D \cap\{\psi: z \notin \operatorname{spt} \psi\}
$$

and define

$$
W_{\psi}(z)=\int_{C} \operatorname{grad} \psi(x) \cdot \frac{x-z}{|x-z|^{m}} d x, \quad \psi \in D(z) .
$$

If it is necessary to specify $C$ we write $W_{\psi}^{C}$ instead of $W_{\psi}$. In case $C$ has a smooth boundary with exterior normal $n$ the integral (2.1) reduces to

$$
\int_{B} \psi(y) \frac{(y-z) \cdot n(y)}{|y-z|^{m}} d H_{m-1}(y),
$$

which is the classical double-layer potential. If $\psi$ vanishes in some neighborhood of $B$ then there is a $Q \subset R^{m}$ with a smooth boundary such that

$$
\text { spt } \psi \cap C \subset \text { int } Q, \quad \operatorname{cl} Q \subset \text { int } C,
$$

whence

$$
W_{\psi}(z)=W_{\psi}^{Q}(z)=0 .
$$

If $z \notin B$, we use this observation to extend $W_{\psi}(z)$ from $D(z)$ to $D$ defining

$$
W_{\nu}(z)=W_{\bar{v}}(z),
$$

where $\bar{\psi}$ is an arbitrary function in $D(z)$ coinciding with given $\psi \in D$ in some neighborhood of $B . W_{\psi}(z)$ may thus be considered as a distribution over $D$ with support in $B$ (compare [D, Chapter III, p. 157]). 
For fixed $\psi \in D, W_{\psi}(z)$ is a harmonic function of $z$ in $R^{m}-B$. Indeed, if $O$ is an open set with $B \cap \operatorname{cl} O=\varnothing$, then there is a $\psi \in D$ coinciding with $\psi$ in some neighborhood of $B$ and vanishing on $O$; clearly,

$$
W_{\psi}(z)=W_{\bar{\psi}}(z)=\int_{C-0} \operatorname{grad} \psi(x) \cdot \frac{x-z}{|x-z|^{m}} d x
$$

is a harmonic function of $z$ in $O$.

Our main objective in this paragraph is to find necessary and sufficient geometric conditions on $C$ securing natural extendability of $W_{\Downarrow}$ from $D$ to broader class of continuous functions and also "nice behaviour" (e.g., boundedness) of $W_{\psi}$ near $B$ for each continuous $\psi$.

2.2. Lemma. Fix $z \in R^{m}$. Then

$$
v_{\infty}^{c}(z)<\infty
$$

is a necessary and sufficient condition to secure

$$
\lim _{k \rightarrow \infty} W_{v_{k}}(z)=W_{\downarrow}(z)
$$

for every sequence of $\psi_{k} \in D(z)$ converging uniformly (as $\left.k \rightarrow \infty\right)$ to $\psi \in D(z)$. If (2.2) holds then there is a $v_{z} \in C^{*}(B)$ such that

$$
\begin{gathered}
W_{\psi}(z)=\int_{B} \psi(y) d \nu_{z}(y), \quad \psi \in D(z), \\
\nu_{z}(\{z\})=0, \\
\left\|\nu_{z}\right\|=v_{\infty}^{C}(z) .
\end{gathered}
$$

(2.3) together with any of the two conditions (2.4), (2.5) determine $\nu_{z}$ uniquely.

Proof. This follows at once from the equality

$$
v_{\infty}^{C}(z)=\sup \left\{W_{\psi}(z): \psi \in D(z),|\psi| \leqq 1\right\}
$$

established in 1.6.

2.3. REMARK. If $(2.2)$ holds we extend $W \cdots(z)$ defining

$$
W f(z)=\int_{B} f(y) d v_{z}(y)
$$

for any bounded Baire function $f$ on $B$.

In order to present another integral representation for $W f(z)$ we introduce the following.

2.4. Notation. Fix $z \in R^{m}$ and $\theta \in \Gamma$. We put for $t>0$

$$
s(t ; z, \theta)=\sigma(= \pm 1)
$$


if there is a $\delta>0$ such that

$$
z+(t+\sigma \tau) \theta \in R^{m}-C, \quad z+(t-\sigma \tau) \theta \in C
$$

for a.e. $\tau \in(0, \delta)$; otherwise we set $s(t ; z, \theta)=0$.

Clearly, $s(t ; z, \theta) \neq 0$ only if $z+t \theta$ is a hit of $\{z+\tau \theta: \tau>0\}$ on $C$.

2.5. LEMMA. If $v_{\infty}^{c}(z)<\infty$ then

$$
W f(z)=\int_{\Gamma}\left\{\sum_{t>0} f(z+t \theta) s(t ; z, \theta)\right\} d H_{m-1}(\theta)
$$

for any bounded Baire function $f$ on $B$.

Proof. Let $v_{\infty}^{c}(z)<\infty$. If $f \in D(z)$ then

$$
\begin{aligned}
W f(z) & =\int_{C} \operatorname{grad} f(x) \cdot \frac{x-z}{|x-z|^{m}} d x \\
& =\int_{\Gamma}\left\{\int_{C_{\theta}} \partial_{\theta} f(z+t \theta) d t\right\} d H_{m-1}(\theta),
\end{aligned}
$$

where

$$
C_{\theta}=\{t: t>0, z+t \theta \in C\}, \partial_{\theta} f=\theta \cdot \operatorname{grad} f .
$$

Noting that $n_{\infty}^{C}(\theta, z)<\infty$ implies

$$
\int_{C_{\theta}} \partial_{\theta} f(z+t \theta) d t=\sum_{t>0} f(z+t \theta) s(t ; z, \theta)
$$

we obtain (2.7).

If $\left\{f_{k}\right\}$ is a pointwise convergent sequence of functions on $B$ such that, for all $k,\left|f_{k}\right| \leqq K$ and (2.7) holds with $f$ replaced by $f_{k}$, then

$$
\left|\sum_{t>0} f_{k}(z+t \theta) s(t ; z, \theta)\right| \leqq K n_{\infty}^{c}(\theta, z)
$$

a.e. $\left\{H_{m-1}\right\}$ on $\Gamma$ and, by the Lebesgue convergence theorem, (2.7) holds for $f=\lim _{k} f_{k}$ as well.

We conclude that (2.7) is valid for every bounded Baire function $f$ vanishing at $z$; in view of (2.4), vanishing at $z$ is irrelevant.

2.6. Proposition. Let $v_{\infty}^{c}(z)<\infty$. Denote by $K_{z}$ and $L_{z}$ the set of all $\theta \in \Gamma$ for which there is an $\varepsilon=\varepsilon(\theta)>0$ such that

$$
H_{1}(\{z+t \theta: 0<t<\varepsilon\} \cap C)=0
$$

and

$$
H_{1}(\{z+t \theta: 0<t<\varepsilon\}-C)=0
$$


respectively. Then $K_{z}, L_{z}$ are measurable $\left(H_{m-1}\right)$,

$$
H_{m-1}\left(\Gamma-\left(K_{z} \cup L_{z}\right)\right)=0
$$

and $\nu_{z}(B)=H_{m-1}\left(L_{z}\right)$ or $\nu_{z}(B)=-H_{m-1}\left(K_{z}\right)$ according as $C$ is bounded or not. If $\psi \in D$, then

$$
\int_{C} \operatorname{grad} \psi(x) \cdot \frac{x-z}{|x-z|^{m}} d x=W_{\psi}(z)-H_{m-1}\left(L_{z}\right) \psi(z) .
$$

If $Q$ is a convex Borel set, then

$$
\left|\nu_{z}(B \cap Q)\right| \leqq A .
$$

Proof. It is easily seen that

$$
\Gamma \cap\left\{\theta: n_{\infty}^{c}(\theta, z)<\infty\right\} \subset L_{z} \cup K_{z}
$$

whence (2.9) follows at once.

Fix now a $\theta \in \Gamma$ with $n_{\infty}^{c}(\theta, z)<\infty$. Let

$$
t_{1}<\cdots<t_{q}
$$

be all the points $t \in(0, \infty)$ with $s(t ; z, \theta) \neq 0$ (cf. 2.4). Clearly,

$$
s\left(t_{j+1} ; \cdots\right)=-s\left(t_{j} ; \cdots\right), \quad 1 \leqq j<q
$$

and $s\left(t_{1} ; \cdots\right)=1$ or $s\left(t_{1} ; \cdots\right)=-1$ according as $\theta \in L_{z}$ or $\theta \in K_{z}$. If $C$ is bounded, then $s\left(t_{q} ; \cdots\right)=1$, while $s\left(t_{q} ; \cdots\right)=-1$ in the opposite case. We conclude that

$$
\sum(\theta)=\sum_{t>0} s(t ; z, \theta)
$$

almost $\left(H_{m-1}\right)$ equals the characteristic function of $L_{z}$ if $C$ is bounded, while $-\sum(\theta)$ almost equals the characteristic function of $K_{z}$ in the opposite case. Employing (2.7) with $f \equiv 1$ we get the first part of our proposition.

Let now $f$ be the characteristic function of a convex Borel set $Q$. Consider again a fixed $\theta \in \Gamma, n_{\infty}^{C}(\theta, z)<\infty$, and the corresponding sequence (2.12). If $t_{i}$ and $t_{k}$ are the first and the last members of (2.12) with $z+t_{j} \theta \in Q$, respectively, then (2.13) implies

$$
\left|\sum_{j=1}^{q} f\left(z+t_{j} \theta\right) s\left(t_{j} ; z, \theta\right)\right|=\left|\sum_{j=i}^{k} s\left(t_{j} ; z, \theta\right)\right| \leqq 1,
$$

whence (2.11) follows by 2.5 . If $\psi \in D$ then we have with the notation from (2.8)

$$
\int_{C_{\theta}} \partial_{\theta} \psi(z+t \theta) d t=\sum_{t>0} \psi(z+t \theta) s(t ; z, \theta)
$$

for $\theta \in K_{z} \cap\left\{\theta: n_{\infty}^{C}(\theta, z)<\infty\right\}$, while

$$
\int_{C_{\theta}} \partial_{\theta} \psi(z+t \theta) d t=\sum_{t>0} \psi(z+t \theta) s(t ; z, \theta)-\psi(z)
$$


for $\theta \in L_{z} \cap\left\{\theta: n_{\infty}^{c}(\theta, z)<\infty\right\}$. Hence

$$
\begin{aligned}
\int_{C} \operatorname{grad} \psi(x) \cdot \frac{x-z}{|x-z|^{m}} d x & =\int_{\Gamma}\left(\int_{C_{\theta}} \partial_{\theta} \psi(z+t \theta) d t\right) d H_{m-1}(\theta) \\
& =W \psi(z)-H_{m-1}\left(L_{z}\right) \psi(z) .
\end{aligned}
$$

2.7. LEMMA. Let $v_{\infty}^{c}(z)<\infty$ and define $L_{z}$ as in 2.6. If $M \subset \Gamma$ is measurable $\left(H_{m-1}\right), H_{m-1}(M)>0$ and

$$
\Lambda_{M}=\{z+t \theta: \theta \in M, t>0\},
$$

then

$$
\lim _{r \rightarrow 0+} \frac{H_{m}\left(\Omega_{r}(z) \cap C \cap \Lambda_{M}\right)}{H_{m}\left(\Omega_{r}(z) \cap \Lambda_{M}\right)}=\frac{H_{m-1}\left(L_{z} \cap M\right)}{H_{m-1}(M)}
$$

In particular, $C$ has an m-dimensional density

$$
d_{C}(z)=H_{m-1}\left(L_{z}\right) / A
$$

at $z$.

Proof. Let $\varepsilon(\theta)$ have the meaning described in the definition of $K_{z}, L_{z}$ in 2.6 and put

$$
\begin{gathered}
K^{r}=M \cap\left\{\theta: \theta \in K_{z}, \varepsilon(\theta)>r\right\}, \\
L^{r}=M \cap\left\{\theta: \theta \in L_{z}, \varepsilon(\theta)>r\right\} .
\end{gathered}
$$

We have

$$
\begin{aligned}
& H_{m}\left(\Omega_{r}(z) \cap C \cap \Lambda_{M}\right) \geqq m^{-1} r^{m} \text { inn } H_{m-1}\left(L^{r}\right), \\
& H_{m}\left(\left(\Omega_{r}(z)-C\right) \cap \Lambda_{M}\right) \geqq m^{-1} r^{m} \text { inn } H_{m-1}\left(K^{r}\right),
\end{aligned}
$$

where inn $H_{m-1}$ stands for the inner $(m-1)$-dimensional Hausdorff measure. Denoting

$$
d_{r}=\frac{H_{m}\left(\Omega_{r}(z) \cap C \cap \Lambda_{M}\right)}{H_{m}\left(\Omega_{r}(z) \cap \Lambda_{M}\right)}
$$

and noting that

$$
K^{r} \uparrow\left(K_{z} \cap M\right), \quad L^{r} \uparrow\left(L_{z} \cap M\right)
$$

as $r \downarrow 0$, we obtain

$$
\begin{gathered}
\liminf _{r \rightarrow 0+} d_{r} \geqq H_{m-1}\left(L_{z} \cap M\right) / H_{m-1}(M), \\
\liminf _{r \rightarrow 0+}\left(1-d_{r}\right) \geqq H_{m-1}\left(K_{z} \cap M\right) / H_{m-1}(M),
\end{gathered}
$$

whence (2.14) follows by (2.9). 
2.8. Notation. $P(C)$ will denote the perimeter of $C$ defined by

$$
P(C)=\sup _{w} \int_{C} \operatorname{div} w(x) d x,
$$

where $w=\left[w_{1}, \ldots, w_{m}\right]$ ranges over all vector-valued functions with $m$ components $w_{j} \in D$ satisfying

$$
\left(\sum_{j=1}^{m} w_{j}^{2}\right)^{1 / 2}=|w| \leqq 1 .
$$

(Further information on sets with finite perimeter may be found in [DG], [F3], [FL], [MA].)

For $M \subset R^{m}$ and $z \in R^{m}$ we let

$$
\operatorname{dist}(z, M)=\inf \{|z-y|: y \in M\} \text {. }
$$

2.9. LEMMA. $v_{\infty}^{C}(z)$ is a lower semicontinuous function of $z$ on $R^{m}$ satisfying the inequality

$$
v_{\infty}^{C}(z) \leqq P(C)(\operatorname{dist}(z, B))^{1-m}, \quad z \notin B .
$$

Proof. If $K<v_{\infty}^{C}(z)$, then there is a $\psi \in D(z)$ such that $|\psi| \leqq 1$ and $W_{\psi}(z)>K$ (see (2.6)). Hence

$$
\liminf _{y \rightarrow z} v_{\infty}^{c}(y) \geqq \lim _{y \rightarrow z} W \psi(y)=W \psi(z)>K .
$$

Suppose now that $z \notin B$, fix an arbitrary $\psi \in D(z)$ with $|\psi| \leqq 1$ and a positive $\rho<\operatorname{dist}(z, B)$. Then there is a $\psi \in D,|\psi| \leqq 1$, which coincides with $\psi$ in some neighborhood of $B$ and vanishes on $\Omega_{\rho}(z)$. Let us define $w(z)=O\left(\in R^{m}\right)$,

$$
w(x)=\psi(x) \frac{x-z}{|x-z|^{m}}, \quad x \neq z
$$

and observe that $|w| \leqq \rho^{1-m}$,

$$
\operatorname{grad} \psi(x) \cdot \frac{x-z}{|x-z|^{m}}=\operatorname{div} w(x)
$$

Consequently,

$$
W \psi(z)=W \bar{\psi}(z)=\int_{C} \operatorname{div} w(x) d x \leqq \rho^{1-m} P(C) .
$$

Remark. We see that $v_{\infty}^{C}(z)$ is finite on $R^{m}-B$ provided $P(C)<\infty$. The converse is also true as it follows from the following

2.10. Proposition. If

$$
\sum_{j=1}^{m+1} v_{\infty}^{C}\left(z_{j}\right)<\infty
$$


for an $(m+1)$-tuple of points $z_{1}, \ldots, z_{m+1}$ in general position (i.e., not situated on a single hyperplane), then

$$
P(C)<\infty \text {. }
$$

Proof. To prove (2.15) it is sufficient to show that

$$
\sup \left\{\int_{C} \partial_{\theta} \psi(x) d x: \psi \in D,|\psi| \leqq 1\right\}<\infty
$$

for every $\theta \in \Gamma$. Fix $\theta \in \Gamma$. Let $\Pi$, denote the hyperplane determined by $\left\{z_{k}: k \neq j\right\}$. Since

$$
\bigcup_{j=1}^{m+1}\left(R^{m}-\Pi_{j}\right)=R^{m}
$$

there are $\alpha_{j} \in D$ such that

$$
\Pi_{j} \cap \operatorname{spt} \alpha_{j}=\varnothing
$$

and

$$
\alpha=\sum_{j=1}^{m+1} \alpha_{j}=1
$$

in some neighborhood of $B$.

Noting that

$$
\int_{C} \alpha(x) \partial_{\theta} \psi(x) d x=\int_{C} \partial_{\theta} \psi(x) d x
$$

we see that it is sufficient to prove that

$$
\sup \left\{\int_{C} \alpha_{j}(x) \partial_{\theta} \psi(x) d x: \psi \in D,|\psi| \leqq 1\right\}<\infty
$$

for $j=1, \ldots, m+1$. Consider, for instance, $j=1$. If $x \in \operatorname{spt} \alpha_{1}$, then $x-z_{2}, \ldots$, $x-z_{m+1}$ are linearly independent. Consequently,

$$
\theta=\sum_{k=2}^{m+1} a_{k}(x) \frac{x-z_{k}}{\left|x-z_{k}\right|^{m}}
$$

where $a_{k}$ are infinitely differentiable in some neighborhood of spt $\alpha_{1}$. Extending $a_{k}$ arbitrarily to $R^{m}$ we get

$$
\int_{C} \alpha_{1}(x) \partial_{\theta} \psi(x) d x=\sum_{k=2}^{m+1} \int_{C} \alpha_{1}(x) a_{k}(x) \operatorname{grad} \psi(x) \cdot \frac{x-z_{k}}{\left|x-z_{k}\right|^{m}} d x .
$$

Fix $k \in\langle 2, m+1\rangle$ and define $F(x)=\alpha_{1}(x) a_{k}(x)$. Then $F \in D\left(z_{k}\right)$ and denoting $K=\max |F|$ we obtain for any $\psi \in D$ with $|\psi| \leqq 1$

$$
\int_{C} F(x) \operatorname{grad} \psi(x) \cdot \frac{x-z_{k}}{\left|x-z_{k}\right|^{m}} d x=I_{1}+I_{2}
$$


where

$$
\begin{aligned}
I_{1} & =\int_{C} \operatorname{grad}(F(x) \psi(x)) \cdot \frac{x-z_{k}}{\left|x-z_{k}\right|^{m}} d x \leqq K v_{\infty}^{C}\left(z_{k}\right), \\
I_{2} & =-\int_{C} \psi(x) \operatorname{grad} F(x) \cdot \frac{x-z_{k}}{\left|x-z_{k}\right|^{m}} d x \\
& \leqq \int_{C}|\operatorname{grad} F(x)| \cdot\left|x-z_{k}\right|^{1-m} d x<\infty .\left({ }^{2}\right)
\end{aligned}
$$

2.11. RemarK. It follows from $2.2,2.9$, and 2.10 that $(2.12)$ is a necessary and sufficient condition to secure continuous dependence (with respect to uniform convergence) of $W \psi(z)$ on $\psi$ for every $z \notin B$. For this reason we agree to impose (2.15) on $C$ throughout the rest of the present paragraph.

Let us recall that $\theta \in \Gamma$ is called the exterior normal of $C$ at $y$ in the sense of Federer provided the symmetric difference of $C$ and the half-space

$$
R^{m} \cap\{x:(x-y) \cdot \theta<0\}
$$

has $m$-dimensional density 0 at $y$ (cf. [F1]).

In what follows the term exterior normal is always to be interpreted in this sense. We put $n^{C}(y)=n(y)=\theta$ if $\theta$ is the exterior normal of $C$ at $y$; otherwise $n(y)$ denotes the zero vector. The set of all $y$ with $n(y) \neq 0$ is called the reduced boundary of $C$ and will be denoted by $\hat{B}$. It is known from [DG2] and [F3] that

$$
H_{m-1}(\hat{B})<\infty
$$

and

$$
\int_{C} \operatorname{div} w(x) d x=\int_{B} w(y) \cdot n(y) d H_{m-1}(y)
$$

for every vector-valued function $w=\left[w_{1}, \ldots, w_{m}\right]$ with components $w_{j} \in D$.

2.12. LeMMA. For every $z \in R^{m}$

$$
v_{\infty}^{C}(z)=\int_{B} \frac{|n(y) \cdot(y-z)|}{|y-z|^{m}} d H_{m-1}(y) .
$$

If $v_{\infty}^{C}(z)<\infty$ and $M \subset B$ is a Borel set, then

$$
\nu_{z}(M)=\int_{M} \frac{n(y) \cdot(y-z)}{|y-z|^{m}} d H_{m-1}(y) .
$$

Proof. Fix $z \in R^{m}$. Let $\psi \in D(z)$ and put $w(z)=O\left(\in R^{m}\right)$,

$$
w(x)=\psi(x) \frac{x-z}{|x-z|^{m}}, \quad x \neq z .
$$

$\left.{ }^{2}\right)$ The author is indebted to Herbert Federer for simplification of this proof. 
Then

$$
W_{\psi}(z)=\int_{C} \operatorname{div} w(x) d x=\int_{B} \psi(y) \frac{n(y) \cdot(y-z)}{|y-z|^{m}} d H_{m-1}(y)
$$

and (2.16) follows from (2.6). Let now $v_{\infty}^{C}(z)<\infty$. As we have just seen,

$$
\int_{B} f d v_{z}=\int_{B} f(y) \frac{n(y) \cdot(y-z)}{|y-z|^{m}} d H_{m-1}(y)
$$

provided $f \in D(z)$; it is easily seen that this formula extends to any bounded Baire function $f$.

The following result will be useful below:

2.13. THEOREM. Let

$$
V^{C}=\sup \left\{v_{\infty}^{C}(y): y \in B\right\}
$$

Then $v_{\infty}^{C}(z) \leqq A+V^{C}$ for every $z \in R^{m}$.

Proof. We may assume $V^{c}<\infty$. Fix $z \in R^{m}-B$ and let $d$ be an arbitrary number less than $v_{\infty}^{C}(z)$. Then there exist mutually disjoint closed parallelepipeds $K_{1}, \ldots, K_{q}$ such that

$$
\sum_{j=1}^{q}\left|\nu_{z}\left(B \cap K_{j}\right)\right|>d
$$

Put $\sigma_{j}=\operatorname{sign} \nu_{z}\left(B \cap K_{f}\right)$ and consider the function

$$
h(x)=\sum_{j=1}^{q} \sigma_{j} \nu_{x}\left(B \cap K_{j}\right),
$$

which is harmonic on

$$
R^{m}-\bigcup_{j=1}^{q} B \cap K_{j} \supset R^{m}-B .
$$

Fix an arbitrary $y \in B$. If $y \notin \bigcup_{j=1}^{q} K_{j}$, then

$$
\lim _{x \rightarrow y} h(x)=h(y) \leqq\left\|\nu_{y}\right\| \leqq V^{c} .
$$

In the opposite case we may assume that $y \in K_{1}$, so that

$$
\lim _{x \rightarrow y} \sum_{j=2}^{q} \sigma_{j} \nu_{x}\left(B \cap K_{j}\right)=\sum_{j=2}^{q} \sigma_{j} \nu_{y}\left(B \cap K_{j}\right) \leqq\left\|\nu_{y}\right\| \leqq V^{C}
$$

and, by Proposition 2.6,

$$
\sup _{x}\left|\nu_{x}\left(B \cap K_{1}\right)\right| \leqq A
$$

We see that

$$
\lim _{x \rightarrow y: x \in B} \sup _{x \in B} h(x) \leqq A+V^{C}
$$


Noting that $h(x) \rightarrow 0$ as $|x| \rightarrow \infty$ we conclude that $h \leqq A+V^{C}$ on $R^{m}-B$. In particular, $d<h(z) \leqq A+V^{C}$.

2.14. CoROllaRY. If $r>0$ and $z \in R^{m}$, then

$$
H_{m-1}\left(\Omega_{r}(z) \cap \hat{B}\right) \leqq m(m+1)^{m}\left(A+V^{C}\right) r^{m-1} .
$$

Proof. To prove (2.17) we may clearly assume that $z=0$. Noting that $V^{C}$ is invariant with respect to dilations of $C$ we observe that it is sufficient to establish (2.17) for $r=1$ only. Let $e^{i}$ denote the point in $R^{m}$ all of whose coordinates vanish with the exception of the $i$ th one which is equal to $m+1$. We have then for $\theta \in \Gamma$ and $y \in \Omega=\Omega_{1}(0)$

$$
\sum_{i=1}^{m}\left|\theta \cdot\left(y-e^{i}\right)\right| \geqq 1,
$$

so that

$$
\begin{aligned}
H_{m-1}(\hat{B} \cap \Omega) & \leqq \sum_{i=1}^{m} \int_{B}\left|n(y) \cdot\left(y-e^{i}\right)\right| d H_{m-1}(y) \\
& \leqq(m+1)^{m} \sum_{i=1}^{m} \int_{B} \frac{\left|n(y) \cdot\left(y-e^{i}\right)\right|}{\left|y-e^{i}\right|^{m}} d H_{m-1}(y) \\
& =(m+1)^{m} \sum_{i=1}^{m} v_{\infty}^{C}\left(e^{i}\right) \leqq m(m+1)^{m}\left(A+V^{C}\right)
\end{aligned}
$$

2.15. THEOREM. Let $C(B)$ denote the Banach space of all continuous functions $f$ on $B$ with the norm $\|f\|=\sup |f|$. If $W f$ is bounded on $R^{m}-B$ for every $f \in C(B)$ then

$$
V^{C}<\infty
$$

\section{If}

$$
C_{i}=R^{m} \cap\left\{z: d_{C}(z)=i\right\} \quad(i=0,1)
$$

and (2.18) holds, then Wf is bounded and uniformly continuous on each of the sets $C_{0}, C_{1}$ and

$$
\begin{array}{cc}
\lim _{z \rightarrow y: z \in C_{1}} W f(z)=W f(y)+A\left(1-d_{C}(y)\right) f(y) & \text { for } y \in B \cap \operatorname{cl} C_{1}, \\
\lim _{z \rightarrow y: z \in C_{0}} W f(z)=W f(y)-A d_{C}(y) f(y) & \text { for } y \in B \cap \operatorname{cl~} C_{0}
\end{array}
$$

whenever $f \in C(B)$.

Proof. If $W f(z)=\left\langle f, v_{z}\right\rangle$ is a bounded function of $z$ on $Q \subset R^{m}$ for every $f \in C(B)$ then, by the uniform boundedness principle, $\left\|\nu_{z}\right\|=v_{\infty}^{C}(z)$ is bounded on $Q$. In view of 2.9, $v_{\infty}^{C}(z)$ must be bounded on cl $Q$ as well. For $Q=R^{m}-B$ we get the first part of our theorem. Assume (2.18) and fix $y \in B$. If $f \equiv 1$ on $B$ then (2.19), 
(2.20) follow from 2.6, 2.7. It is therefore sufficient to prove (2.19), (2.20) assuming $f \in C(B), f(y)=0$. For every $k$ we have the decomposition $f=f_{k}+g_{k}$, where $f_{k} \in C(B)$ vanishes in some neighborhood of $y$ in $B$ and $\left\|g_{k}\right\| \leqq 1 / k$. Then $W f_{k}$ is continuous at $y$ and $\left|W g_{k}\right| \leqq\left(A+V^{C}\right) / k$. We see that $W f=\lim _{k \rightarrow \infty} W f_{k}$ is continuous at $y$. The rest is obvious.

\section{The Fredholm radius of an operator.}

3.1. Notation. As in the introduction, $G$ will stand for a fixed open set with a compact boundary $B$ in $R^{m}$. We put $C=R^{m}-G$ and write $v_{r}(y)=v_{r}^{G}(y)\left(=v_{r}^{C}(y)\right)$, $V=V^{C}$ (cf. 1.6, 1.7, (1.12), 2.13). We always assume

$$
V<\infty \text {. }
$$

In view of 1.13 ,

$$
N U: \mu \rightarrow N U \mu
$$

is a bounded linear operator on $C^{*}(B)$. By $2.7, G$ has an $m$-dimensional density $d_{G}(y)$ at any $y \in R^{m}$.

\subsection{LemMA. If $f$ is a bounded Baire function on $B$ then}

$$
\left\langle f, N U \delta_{y}\right\rangle=A d_{G}(y) f(y)+W^{c} f(y), \quad y \in B .
$$

Proof. It is sufficient to prove (3.3) for $f \in D$ only. Employing 1.3, 2.6, and 2.7 we obtain

$$
\begin{aligned}
\left\langle f, N U \delta_{y}\right\rangle & =\int_{G} \operatorname{grad} f(x) \cdot \frac{y-x}{|y-x|^{m}} d x \\
& =A f(y)+\int_{C} \operatorname{grad} f(x) \cdot \frac{x-y}{|x-y|^{m}} d x \\
& =A d_{G}(y) f(y)+W^{C} f(y) .
\end{aligned}
$$

3.3. Definition. If $f \in C(B)$ we define

$$
\bar{W} f(y)=\left\langle f, N U \delta_{y}\right\rangle-\frac{1}{2} A f(y), \quad y \in B .
$$

3.4. Lemma. $\bar{W} f \in C(B)$ whenever $f \in C(B)$. The operator

$$
\bar{W}: f \rightarrow \bar{W} f
$$

is bounded on $C(B)$ and the operator (3.2) is adjoint to $\frac{1}{2} A I+\bar{W}$, where I is the identity operator on $C(B)$. If $f \in C(B)$ and $C_{1}$ has the meaning described in 2.15 , then

$$
\begin{aligned}
\bar{W} f(y) & =\lim _{z \rightarrow y ; z \in C_{1}} W^{c} f(z)-\frac{1}{2} A f(y), & & y \in B \cap \mathrm{cl} C_{1}, \\
\bar{W} f(y) & =W^{c} f(y)+A\left(d_{G}(y)-\frac{1}{2}\right) f(y) & & \\
& =\lim _{z \rightarrow y: z \in G} W^{C} f(z)+\frac{1}{2} A f(y), & & y \in B .
\end{aligned}
$$


Proof. (3.7), (3.6) follow from (3.4), (3.3), and (2.19), (2.20). By (3.7), $\bar{W} f \in C(B)$ for $f \in C(B)$. If $\nu_{y}$ has the meaning described in 2.2 and

$$
\bar{\nu}_{y}=A\left(d_{G}(y)-\frac{1}{2}\right) \delta_{y}+\nu_{y},
$$

then

$$
\bar{W} f(y)=\left\langle f, \bar{\nu}_{y}\right\rangle, \quad f \in C(B), \quad y \in B,
$$

whence

$$
\|\bar{W}\|=\sup _{y \in B}\left\|\bar{\nu}_{y}\right\|=\sup _{y \in B}\left(A\left|d_{G}(y)-\frac{1}{2}\right|+v_{\infty}(y)\right) .
$$

By 1.13, the formula (1.2) holds for any $\psi \in C(B)$. This together with (3.4) implies

$$
N U=\left(\frac{1}{2} A I+\bar{W}\right)^{*}
$$

where $(\cdots)$ * denotes the operator adjoint to $(\cdots)$.

3.5. REMARK. In $\$ 4$ we shall be engaged with the Neumann problem in the following formulation: Given $\nu \in C^{*}(B)$ find a $\mu \in C^{*}(B)$ with $N U_{\mu}=\nu$. By (3.11), this problem reduces to solving the equation

$$
\left(\frac{1}{2} A I+\bar{W}\right)^{*} \mu=\nu .
$$

In connection with this equation it is useful to know the Fredholm radius of $\bar{W}$, i.e., the reciprocal of

$$
\omega \bar{W}=\inf _{T}\|\bar{W}-T\|,
$$

where $T$ ranges over all compact operators on $C(B)$ (cf. [RS]). Our main objective in $\S 3$ is to express $\omega \bar{W}$ in terms of geometric quantities connected with $G$ and investigate relations between $\omega \bar{W}$ and regularity of $B$.

3.6. THEOREM. Let $I_{B}$ denote the set of all isolated points of $B$ and put $E=B-I_{B}$ if $I_{B}$ is finite, $E=B$ in the opposite case. Let $V_{r}=0$ or

$$
V_{r}=\sup _{y \in E}\left[A\left|\frac{1}{2}-d_{G}(y)\right|+v_{r}(y)\right]
$$

according as $E=\varnothing$ or not and define

$$
V_{0}=\lim _{r \rightarrow 0+} V_{r}
$$

Then $\omega \bar{W}=V_{0}$.

Proof will be divided into two steps.

Step 1. We first prove that

$$
\omega \bar{W} \leqq V_{r}
$$

for every $r>0$ satisfying

$$
H_{m-1}(\hat{B} \cap\{z:|z-y|=r\})=0 \quad \text { for all } y,
$$


where $B$ is the reduced boundary defined in 2.11 . If $R$ is the set of all $r>0$ enjoying (3.13) then $(0, \infty)-R$ is at most countable, because spherical shells with different radii meet each other in a set of $H_{m-1}$-measure zero and $H_{m-1}(\hat{B})<\infty$. Hence $V_{0}=\inf \left\{V_{r}: r \in R\right\}$ and

$$
\omega \bar{W} \leqq V_{0}
$$

will follow from (3.12). So let us fix $r \in R$. If $I_{B}$ is finite we assume, as we may, $r<\operatorname{dist}\left(I_{B}, E\right)=\inf \left\{\operatorname{dist}(z, E): z \in I_{B}\right\}$. Let $c_{y}$ denote the characteristic function of $B-\left(\Omega_{r}(y) \cap E\right)$ and put

$$
W_{r} f(y)=\int_{B} c_{y} f d \bar{\nu}_{y}, \quad f \in C(B),
$$

where $\bar{\nu}_{y}$ is defined by (3.8). Absolute values of all the functions in

$$
\left\{W_{r} f: f \in C(B),\|f\| \leqq 1\right\}
$$

are bounded by $\sup _{y \in B}\left\|\bar{\nu}_{y}\right\| \leqq \frac{1}{2} A+V$. If $f \in C(B)$ and $x, y$ are arbitrary points in $E$ with $|x-y|=d \leqq \frac{1}{2} r$, then we obtain from 2.12

$$
W_{r} f(x)-W_{r} f(y)=J_{1}(f)+J_{2}(f),
$$

where

$$
\begin{aligned}
& J_{1}(f)=\int_{B} f(z)\left[c_{x}(z)-c_{y}(z)\right] \frac{n(z) \cdot(z-x)}{|z-x|^{m}} d H_{m-1}(z) \\
& J_{2}(f)=\int_{B} f(z) c_{y}(z)\left[\frac{z-x}{|z-x|^{m}}-\frac{z-y}{|z-y|^{m}}\right] \cdot n(z) d H_{m-1}(z)
\end{aligned}
$$

Denoting

$$
\alpha(d)=\sup _{z} H_{m-1}\left[\left(\mathrm{cl} \Omega_{r+d}(z)-\Omega_{r-d}(z)\right) \cap \hat{B}\right]
$$

we get for $\|f\| \leqq 1$

$$
\begin{aligned}
& \left|J_{1}(f)\right| \leqq\left(\frac{1}{2} r\right)^{1-m} \alpha(d), \\
& \left|J_{2}(f)\right| \leqq(m+1) d\left(\frac{1}{2} r\right)^{-m} .
\end{aligned}
$$

Since $r \in R$, an easy compactness argument yields

$$
\lim _{d \rightarrow 0+} \alpha(d)=0 .
$$

We see that all the functions in (3.15) are equicontinuous on $E$; noting that $B-E$ is finite we conclude that they are equicontinuous on $B$ as well and the operator

$$
W_{r}: f \rightarrow W_{r} f
$$

is compact. Hence

$$
\omega \bar{W} \leqq\left\|\bar{W}-W_{r}\right\| .
$$


If $f \in C(B)$ then

$$
W_{r} f(y)=\bar{W} f(y) \quad \text { for } y \in B-E
$$

while (3.9) shows that, for $y \in E$,

$$
\left(\bar{W}-W_{r}\right) f(y)=\int f d \bar{\nu}_{y}
$$

with the integral extended over $B \cap \Omega_{r}(y)$. Consequently,

$$
\left\|\bar{W}-W_{r}\right\|=\sup _{y \in E}\left|\bar{\nu}_{y}\right|\left(\Omega_{r}(y) \cap B\right)=V_{r}
$$

and (3.12) is established.

Step 2. Now we are going to prove the inequality

$$
\omega \bar{W} \geqq V_{0}
$$

which is trivial if $E=\varnothing$. Therefore we assume $E \neq \varnothing$, so that $E$ is infinite. A point $y \in B$ will be termed a discontinuity for a $\mu \in C^{*}(B)$ if $\mu(\{y\}) \neq 0$. By the Radon theorem, every compact operator on $C(B)$ can be arbitrarily closely approximated by operators of finite rank. If $Q$ is such an operator, sending $f \in C(B)$ into

$$
Q f=\sum_{k=1}^{q} g_{k}\left\langle f, m_{k}\right\rangle,
$$

where $g_{k} \in C(B)$ and $m_{k} \in C^{*}(B)$, then every $m_{k}$ can be arbitrarily closely (in the norm of $C^{*}(B)$ ) approximated by $\bar{m}_{k} \in C^{*}(B)$ having only a finite number of discontinuities. Defining

$$
\bar{Q} \cdots=\sum_{k=1}^{q} g_{k}\left\langle\cdots, \bar{m}_{k}\right\rangle
$$

we see that the deviation $\|Q-\bar{Q}\|$ can be made as small as we want. It follows from these observations that, in order to prove (3.16), it is sufficient to show that

$$
\|\bar{W}-Q\| \geqq V_{0}
$$

for every $Q$ of the type (3.17), where $m_{k} \in C^{*}(B)$ have only a finite number of discontinuities each. Let us fix such a $Q$ and denote by $K$ the (finite) set of all $y \in B$ which represent a discontinuity for some of the measures $m_{k}(k=1, \ldots, q)$. Every $m_{k}$ splits into $m_{k}^{1}$ having no discontinuities and a finite combination of Dirac measures, to be denoted by $m_{k}^{2}$. Since $y$ is the only possible discontinuity for $\bar{\nu}_{y}$, we have for $y \in B-K$

$$
\left\|\bar{\nu}_{y}-\sum_{k} g_{k}(y) m_{k}\right\|=\left\|\bar{\nu}_{y}-\sum_{k} g_{k}(y) m_{k}^{1}\right\|+\left\|\sum_{k} g_{k}(y) m_{k}^{2}\right\|
$$

whence

$$
\|\bar{W}-Q\| \geqq \sup \left\{\left\|\bar{\nu}_{y}-\sum_{k} g_{k}(y) m_{k}^{1}\right\|: y \in E-K\right\}
$$


Since the operator

$$
f \rightarrow\left\langle f, \bar{\nu}_{y}-\sum_{k} g_{k}(y) m_{k}^{1}\right\rangle
$$

sends each $f \in C(B)$ into a continuous function of $y$ we conclude that

$$
a_{r}(y)=\left|\vec{\nu}_{y}-\sum_{k} g_{k}(y) m_{k}^{1}\right|\left(\Omega_{r}(y) \cap B\right)
$$

is a lower semicontinuous function of $y$ for every $r>0$. Consequently,

$$
\begin{aligned}
\|\bar{W}-Q\| & \geqq \sup \left\{a_{r}(y): y \in E-K\right\} \\
& =\sup \left\{a_{r}(z): z \in E-\left(I_{B} \cap K\right)\right\} .
\end{aligned}
$$

Consider now an arbitrary $y \in E \cap I_{B} \cap K$ and note that $E \cap I_{B} \neq \varnothing$ implies

$$
\varnothing \neq E \cap\left(I_{B}-K\right) \subset E-\left(I_{B} \cap K\right) .
$$

If

$$
r<\operatorname{dist}\left(I_{B} \cap K, E-\left(I_{B} \cap K\right)\right)
$$

then

$$
\begin{aligned}
\left|\sum_{k} g_{k}(y) m_{k}^{1}\right|\left(\Omega_{r}(y) \cap B\right) & =\left|\sum_{k} g_{k}(y) m_{k}^{1}\right|\left(\Omega_{r}(y) \cap I_{B}\right)=0, \\
a_{r}(y) & =\left|\bar{\nu}_{y}\right|\left(\Omega_{r}(y) \cap I_{B}\right)=\frac{1}{2} A .
\end{aligned}
$$

On the other hand, we have for any $z \in I_{B}$

$$
\frac{1}{2} A \leqq\left|A\left(\frac{1}{2}-d_{G}(z) \delta_{z}\right)\right|\left(\Omega_{r}(z) \cap B\right)+\left|\nu_{z}-\sum_{k} g_{k}(z) m_{k}^{1}\right|\left(\Omega_{r}(z) \cap B\right)=a_{r}(z),
$$

because $\nu_{z}-\sum_{k} g_{k}(z) m_{k}^{1}$ has no discontinuities. Combining this with (3.20) we get

$$
a_{r}(y) \leqq \sup \left\{a_{r}(z): z \in E \cap\left(I_{B}-K\right)\right\} \leqq \sup \left\{a_{r}(z): z \in E-\left(I_{B} \cap K\right)\right\} .
$$

We have thus for small $r>0$

$$
\sup \left\{a_{r}(z): z \in E-\left(I_{B} \cap K\right)\right\}=\sup \left\{a_{r}(y): y \in E\right\} .
$$

Note that

$$
V_{r}=\sup _{y \in E}\left|\bar{\nu}_{y}\right|\left(\Omega_{r}(y) \cap B\right) .
$$

If $M=\max \left\{\left|g_{k}(x)\right|: x \in B, 1 \leqq k \leqq q\right\}$, then

$$
\sup \left\{a_{r}(y): y \in E\right\} \geqq V_{r}-M \sum_{k} \sup _{y \in E}\left|m_{k}^{1}\right|\left(\Omega_{r}(y) \cap B\right) .
$$


Since $m_{k}^{1}(k=1, \ldots, q)$ have no discontinuities,

$$
\lim _{r \rightarrow 0+} \sup _{y \in B}\left|m_{k}^{1}\right|\left(\Omega_{r}(y) \cap B\right)=0 .
$$

Making $r \rightarrow 0+$ in (3.22) and using (3.21), (3.19) we arrive at (3.18).

REMARK. The basic idea of the above proof goes back to J. Radon (cf. [RS]).

3.7. Lemma. Let us define $\hat{B}$ as in 2.11 and put

$$
B^{*}=B \cap\left\{y:\left|d_{G}(y)-\frac{1}{2}\right|<\frac{1}{2}\right\} \text {. }
$$

Then $\hat{B}$ is dense in $B^{*}$ (moreover, every ball of center in $B^{*}$ meets $\hat{B}$ in a set of positive $H_{m-1}$-measure) and

$$
H_{m-1}\left(B^{*}-\widehat{B}\right)=0 .
$$

Proof. If $y \in B^{*}$ then there is an $\varepsilon>0$ such that

$$
\begin{gathered}
H_{m}\left(\Omega_{r}(y) \cap G\right)>\varepsilon H_{m}\left(\Omega_{r}(y)\right), \\
H_{m}\left(\Omega_{r}(y) \cap C\right)>\varepsilon H_{m}\left(\Omega_{r}(y)\right)
\end{gathered}
$$

for $0<r<\varepsilon$. By the relative isoperimetric inequality for sets with finite perimeter (cf. Theorem (4.3) in [MI]; general isoperimetric inequalities for currents may be found in $[\mathrm{FF}, \S 6])$ we conclude that

$$
H_{m-1}\left(\Omega_{r}(y) \cap \hat{B}\right) \geqq \alpha r^{m-1}, \quad 0<r<\varepsilon,
$$

where $\alpha>0$ does not depend on $r$. Hence it follows by [F2, §3] that

$$
H_{m-1}\left(B^{*}-\hat{B}\right)=0 \text {. }
$$

3.8. Notation. For $z \in R^{m}, r>0$ and $\theta \in \Gamma$ we put

$$
\Omega_{r}(z, \theta)=\Omega_{r}(z) \cap\{x:(x-z) \cdot \theta>0\} .
$$

We denote by $a(\theta, \eta)=\arccos (\theta \cdot \eta)$ the nonoriented angle enclosed by $\theta, \eta \in \Gamma$. It is easily seen that

$$
\frac{a(\theta, \eta)}{2 \pi}=\frac{H_{m}\left(\Omega_{r}(z, \theta) \cap \Omega_{r}(z,-\eta)\right)}{H_{m}\left(\Omega_{r}(z)\right)}
$$

The symbol $n$ will always have the meaning described in 2.11. The symmetric difference of $P, Q \subset R^{m}$ will be denoted by $P-Q$.

3.9. LemMA. Let $z \in \hat{B}, \theta=n(z)$. Then

$$
H_{m}\left(\Omega_{r}(z, \theta) \cap \text { int } C\right)+H_{m}\left(\Omega_{r}(z,-\theta) \cap G\right) \leqq H_{m}\left(\Omega_{r}(z)\right) \frac{v_{r}(z)}{A} .
$$

Proof. Let

$$
\gamma_{1}<\frac{H_{m}\left(\Omega_{r}(z, \theta) \cap \text { int } C\right)}{H_{m}\left(\Omega_{r}(z)\right)}, \quad \gamma_{2}<\frac{H_{m}\left(\Omega_{r}(z,-\theta) \cap G\right)}{H_{m}\left(\Omega_{r}(z)\right)} .
$$


Put $\Gamma_{+}=\Gamma \cap\{\eta: \eta \cdot \theta>0\}, \Gamma_{-}=\Gamma \cap\{\eta: \eta \cdot \theta<0\}, S(\rho)=\{x:|x-z|=\rho\}$ and define $K_{z}, L_{z}$ as in 2.6. There are $\rho_{1}, \rho_{2} \in(0, r)$ such that

$$
\begin{aligned}
& H_{m-1}\left(S\left(\rho_{1}\right) \cap \Omega_{r}(z, \theta) \cap \operatorname{int} C\right)>\gamma_{1} H_{m-1}\left(S\left(\rho_{1}\right)\right), \\
& H_{m-1}\left(S\left(\rho_{2}\right) \cap \Omega_{r}(z,-\theta) \cap G\right)>\gamma_{2} H_{m-1}\left(S\left(\rho_{2}\right)\right) .
\end{aligned}
$$

By virtue of 2.7

$$
\begin{aligned}
& H_{m-1}\left(L_{z} \cap \Gamma_{+}\right)=\frac{1}{2} A \lim _{\rho \rightarrow 0+} \frac{H_{m}\left(\Omega_{\rho}(z, \theta) \cap C\right)}{H_{m}\left(\Omega_{\rho}(z, \theta)\right)}=0, \\
& H_{m-1}\left(L_{z} \cap \Gamma_{-}\right)=\frac{1}{2} A \lim _{\rho \rightarrow 0+} \frac{H_{m}\left(\Omega_{\rho}(z,-\theta) \cap C\right)}{H_{m}\left(\Omega_{\rho}(z,-\theta)\right)}=\frac{1}{2} A .
\end{aligned}
$$

We see that $L_{z}$ is equivalent $\left(H_{m-1}\right)$ with $\Gamma_{-}$and $K_{z}$ is equivalent $\left(H_{m-1}\right)$ with $\Gamma_{+}$. If $\eta \in L_{z}$ and $\{z+\rho \eta: 0<\rho<r\} \cap G \neq \varnothing$ then, with the notation from 1.6, $n_{r}(\eta, z) \geqq 1$. Employing (3.25) we obtain

$$
\int_{L_{z}} n_{r}(\eta, z) d H_{m-1}(\eta)>\gamma_{2} A
$$

Similarly, (3.24) implies

$$
\int_{K_{z}} n_{r}(\eta, z) d H_{m-1}(\eta)>\gamma_{1} A
$$

so that

$$
v_{r}(z)=\int_{\Gamma} n_{r}(\eta, z) d H_{m-1}(\eta)>\left(\gamma_{1}+\gamma_{2}\right) A
$$

3.10. LEMMA. Let $N \in \Gamma, y \in R^{m}, r>0$ and suppose that

$$
\begin{gathered}
\sup _{z \in B} v_{r}(z) \leqq u_{0} A, \\
H_{m}\left(\Omega_{r}(y, N) \cap C\right) \leqq u_{1} H_{m}\left(\Omega_{r}(y)\right), \\
H_{m}\left(\Omega_{r}(y,-N) \cap \operatorname{cl} G\right) \leqq u_{2} H_{m}\left(\Omega_{r}(y)\right) .
\end{gathered}
$$

If $s=u_{0}+u_{1}+u_{2}<\frac{1}{2}$, then for every $\gamma>s$ there is $a \delta>0$ (depending on $(\gamma-s) r$ only) such that

$$
a(n(z), N) \leqq \pi \gamma \quad \text { for } z \in \hat{B} \cap \Omega_{\delta}(y) .
$$

Proof. Let $\gamma=s+2 \varepsilon, \varepsilon>0$, and consider a $\theta \in \Gamma$ with $a(N, \theta)>\gamma \pi$. We have by (3.23)

$$
\begin{aligned}
& H_{m}\left(\Omega_{r}(y,-N) \cap \Omega_{r}(y, \theta)\right)>\frac{1}{2} \gamma H_{m}\left(\Omega_{r}(y)\right), \\
& H_{m}\left(\Omega_{r}(y, N) \cap \Omega_{r}(y,-\theta)\right)>\frac{1}{2} \gamma H_{m}\left(\Omega_{r}(y)\right) .
\end{aligned}
$$


Let us fix $\delta>0$ small enough to secure

$$
H_{m}\left(\Omega_{r}(z, \eta)-\Omega_{r}(y, \eta)\right)<\varepsilon H_{m}\left(\Omega_{r}(y)\right)
$$

for $|z-y|<\delta$ and any $\eta \in \Gamma$. We have then for $z \in \Omega_{\delta}(y)$

$$
\begin{aligned}
& H_{m}\left(\Omega_{r}(y,-N) \cap \Omega_{r}(z, \theta)\right)>\frac{1}{2} s H_{m}\left(\Omega_{r}(z)\right), \\
& H_{m}\left(\Omega_{r}(y, N) \cap \Omega_{r}(z,-\theta)\right)>\frac{1}{2} s H_{m}\left(\Omega_{r}(z)\right),
\end{aligned}
$$

whence we obtain on account of (3.27), (3.28)

$$
\begin{aligned}
& H_{m}\left(\Omega_{r}(z, \theta) \cap \text { int } C\right) \geqq H_{m}\left(\Omega_{r}(z, \theta) \cap \Omega_{r}(y,-N)\right)-H_{m}\left(\Omega_{r}(y,-N) \cap \mathrm{cl} G\right) \\
&>\left(\frac{1}{2} s-u_{2}\right) H_{m}\left(\Omega_{r}(z)\right), \\
& H_{m}\left(\Omega_{r}(z,-\theta)\right.\cap G) \geqq H_{m}\left(\Omega_{r}(z,-\theta) \cap \Omega_{r}(y, N)\right) \\
&-H_{m}\left(\Omega_{r}(y, N) \cap C\right)>\left(\frac{1}{2} s-u_{1}\right) H_{m}\left(\Omega_{r}(z)\right) .
\end{aligned}
$$

Suppose now that $z \in \hat{B}$ and $\theta=n(z)$. Employing (3.29), (3.30) and Lemma 3.9 we arrive at $v_{r}(z)>u_{0} A$, which violates (3.26).

3.11. Notation. Let $P_{N}$ stand for the orthogonal projection of $R^{m}$ onto $R^{m} \cap\{x: x \cdot N=0\}$. With every $\alpha \in\left(0, \frac{1}{2}\right)$ we associate $B(\alpha) \subset B$ as follows. We let $y \in B(\alpha)$ if for every $\gamma \in\left(\alpha, \frac{1}{2}\right)$ there is a neighborhood $Q$ of $y$ in $B$ and an $N \in \Gamma$ such that $\left|P_{N}(x)-P_{N}(z)\right| \geqq|x-z| \cos \pi \gamma$ whenever $x, z \in Q$. By Theorem 5.1 in [MI] we get the following corollary of Lemma 3.10:

3.12. CoROllaRY. If (3.26), (3.27), (3.28) hold and $s=u_{0}+u_{1}+u_{2}<\frac{1}{2}$, then $y \in B(s)$; moreover, for every $\gamma \in\left(s, \frac{1}{2}\right)$ there is a $\delta>0$ (depending on $r(\gamma-s)$ only) such that $B \cap \Omega_{\delta}(y) \subset B(\gamma)$.

3.13. THEOREM. If $V_{0}<\frac{1}{2} A$ then $I_{B}$ is finite and

$$
H_{m-1}\left(B-B\left(V_{0} / A\right)\right)=0 .
$$

If $V_{0}<\frac{1}{4} A$ then $B=B\left(2 V_{0} / A\right)$.

Proof. Let $V_{0}<\frac{1}{2} A$. Then $I_{B}$ must be finite, $B-I_{B}=E \subset B^{*}$ and, by 3.7, $H_{m-1}(B-B)=0$. To prove (3.31) it is therefore sufficient to show that

$$
\hat{B} \subset B\left(3 \varepsilon+\frac{V_{0}}{A}\right)
$$

for every small $\varepsilon>0$. Fix $y \in \hat{B}, N=n(y)$ and $\varepsilon>0,3 \varepsilon+V_{0} / A<\frac{1}{2}$. We have then for sufficiently small $r>0$

$$
\begin{aligned}
H_{m}\left(\Omega_{r}(y, N) \cap C\right) & \leqq \varepsilon H_{m}\left(\Omega_{r}(y)\right), \\
H_{m}\left(\Omega_{r}(y,-N) \cap \operatorname{cl} G\right) & =H_{m}\left(\Omega_{r}(y,-N) \cap G\right) \\
& \leqq \varepsilon H_{m}\left(\Omega_{r}(y)\right), \\
V_{r} & <V_{0}+\varepsilon A .
\end{aligned}
$$


Employing 3.12 we get $y \in B\left(3 \varepsilon+V_{0} / A\right)$. Suppose now that $\alpha=V_{0} / A<\frac{1}{4}$ and fix an $r>0$ with (3.32). By 3.9 we have for all $y \in \hat{B}$

$$
H_{m}\left(\Omega_{r}(y, n(y)) \cap C\right)+H_{m}\left(\Omega_{r}(y,-n(y)) \cap \mathrm{cl} G\right)<(\alpha+\varepsilon) H_{m}\left(\Omega_{r}(y)\right) .
$$

By 3.12 there is a $\delta>0$ independent of $y$ such that $\Omega_{\delta}(y) \cap B \subset B(3 \varepsilon+2 \alpha)$ for every $y \in \hat{B}$. It remains to note that $\hat{B}$ is dense in $E$ by 3.7 .

\subsection{Corollary. If $V_{0}<\frac{1}{4} A$ then}

$$
\lim _{r \rightarrow 0+} \sup \left\{\rho^{1-m} H_{m-1}\left(\Omega_{\rho}(y) \cap B\right): y \in B, 0<\rho<r\right\} \leqq b_{m-1} \sec \left(2 V_{0} \pi / A\right),
$$

where $b_{m-1}$ denotes the volume of the unit ball in $R^{m-1}$.

3.15. THEOREM. Let $V_{0}=0$ (which means that $\bar{W}$ is compact). Then $I_{B}$ is finite and $E=B-I_{B}$ is a surface of class $C^{1}$.

Proof. For every $\varepsilon>0, \varepsilon<\frac{1}{4}$ there is an $r>0$ such that $V_{r}<\varepsilon A$. By 3.9 (note also that $H_{m}(B)=0$ )

$$
H_{m}\left(\Omega_{r}(y, n(y)) \cap C\right)+H_{m}\left(\Omega_{r}(y,-n(y)) \cap \mathrm{cl} G\right)<\varepsilon H_{m}\left(\Omega_{r}(y)\right)
$$

for all $y \in \hat{B}$. Employing 3.10 we get a $\delta>0$ depending on $r \varepsilon$ only such that, for every couple of points $y, z \in \hat{B}, a(n(z), n(y)) \leqq 3 \varepsilon \pi$ whenever $|y-z|<\delta$. We see that $n$ is uniformly continuous on $\hat{B}$. By $3.7, n$ extends to a continuous function $N$ on $E=\operatorname{cl} \hat{B}$ and, for every $y \in E$,

$$
N(y)=\lim _{r \rightarrow 0+} \frac{\int N(z) d H_{m-1}(z)}{H_{m-1}\left(\Omega_{r}(y) \cap E\right)}
$$

with the integral extended over $\Omega_{r}(y) \cap E$. Hence it follows by [DG3, Theorem III] (see also definition of the reduced boundary presented in [DG3, p. 10]) that $E$ is a surface of class $C^{1}$.

REMARK. The main results of this paper (such as Theorem 1.13 or Theorem 3.6) are expressed in terms of the quantity $v_{r}(y)$. In the definition of $v_{r}(y)$ one considers all half-lines issuing at $y$, i.e., orthogonal trajectories of the level surfaces of the Green function with a fixed pole at $y$. This suggests the possibility of generalizing these results to the case of a Green space in the sense of [BC].

\section{Boundary value problems.}

Notation. We shall keep the notation and assumptions introduced in $\$ 3$. Besides that we always assume here that $m>2$ (see Remark 4.10 below dealing with $m=2)$. We shall start with investigation of solutions of the equations

$$
\begin{aligned}
\left(\frac{1}{2} A I+\bar{W}\right) f=0 & \text { over } C(B), \\
\left(\frac{1}{2} A I+\bar{W}\right)^{*} \mu=0 & \text { over } C^{*}(B) .
\end{aligned}
$$

$C_{0}(B)$ will denote the class of all $f \in C(B)$ satisfying (4.1) and $C^{*}(B)$ will stand for 
the set of all $\mu \in C^{*}(B)$ satisfying (4.2). We agree to use $M$ as a generic notation for a Borel set. If $\mu$ is a signed Borel measure in $R^{m}$ and $R \subset R^{m}$ is a fixed Borel set, we define $\mu \cap R$ by

$$
\mu \cap R(M)=\mu(M \cap R), \quad M \subset R^{m} .
$$

Recalling the definition of $\bar{\nu}_{y}$ presented in (3.8) we obtain from (3.9) that, for every $\mu \in C^{*}(B)$,

$$
\bar{W}^{*} \mu(M)=\int_{B} \bar{\nu}_{y}(M) d \mu(y), \quad M \subset B .
$$

It follows from (3.10) that

$$
\left\|W^{*}\right\| \leqq \frac{1}{2} A+V
$$

where $V=V^{C}$ has been defined in 2.13 .

4.1. Lemma. If $\mu \in C_{0}^{*}(B)$ then $|\mu|\left(I_{B}\right)=0$ (see 3.6 for notation).

Proof. Let $\mu \in C_{0}^{*}(B), z \in I_{B}$ and denote by $f$ the characteristic function of $\{z\}$. We have by $3.4,1.13$, and (3.3)

$$
0=N U \mu(\{z\})=\int_{B}\left(A d_{G}(y) f(y)+W^{C} f(y)\right) d \mu(y) .
$$

It follows from (2.12) that $W^{c} f=0$, so that $A d_{G}(y) f(y)+W^{c} f(y)=A f(y)$ for all $y \in B$. Hence $\mu(\{z\})=0$.

REMARK. A refinement of the preceding argument may be used to show that, for every $\mu \in C_{0}^{*}(B), \mu \cap M$ is absolutely continuous with respect to $H_{m-1} \cap \hat{B}$ provided $d_{G}(y)>0$ for all $y \in M$.

As it follows from $4.1, C_{0}^{*}(B)$ contains only trivial measure in case $B=I_{B}$. In what follows we always exclude the trivial case of a finite $B$.

4.2. Lemma. Fix $z \in B, \mu \in C^{*}(B)$ and put for $t>0$

$$
\begin{aligned}
R_{t} & =B \cap \Omega_{t}(z), \\
\alpha(t) & =H_{m-1}\left(R_{t} \cap \hat{B}\right), \\
\beta(t) & =|\mu|\left(R_{t}\right) .
\end{aligned}
$$

Let $0<\rho<\delta<\Delta$ and suppose that

$$
\mu \cap\left(R_{\Delta}-R_{\delta}\right)=\mu .
$$

Then

$$
\left|\bar{W}^{*} \mu\right|\left(R_{\rho}\right) \leqq \frac{\alpha(\rho) \beta(\Delta)}{(\Delta-\rho)^{m-1}}+(m-1) \alpha(\rho) \int_{\delta}^{\Delta} \frac{\beta(t) d t}{(t-\rho)^{m}}
$$


Proof. Let $g$ denote the, characteristic function of $\hat{B} \cap R_{\rho}$. By 2.12 we obtain for $y \in R_{\Delta}-R_{\delta}$ and $M \subset R_{\rho}$

$$
\left|\bar{\nu}_{y}(M)\right|=\left|\nu_{y}(M)\right| \leqq \int_{M} g(x)|y-x|^{1-m} d H_{m-1}(x)
$$

whence it follows easily by (4.3)

$$
\left|\bar{W}^{*} \mu\right|\left(R_{\rho}\right) \leqq \iint_{B \times B} g(x)|y-x|^{1-m} d H_{m-1}(x) d|\mu|(y) .
$$

Since

$$
\begin{aligned}
\int_{B}|y-x|^{1-m} d|\mu|(y) & \leqq \int_{\delta}^{\Delta}(t-|x|)^{1-m} d \beta(t) \\
& \leqq \frac{\beta(\Delta)}{(\Delta-|x|)^{m-1}}+(m-1) \int_{\delta}^{\Delta} \frac{\beta(t) d t}{(t-|x|)^{m}}
\end{aligned}
$$

(4.9) implies (4.8).

4.3. LEMMA. Fix $z \in B, r>0$ and put, with the notation from 4.2,

$$
\begin{aligned}
R & =R_{r}\left(=\Omega_{r}(z) \cap B\right) \\
V(R) & =\sup \left\{\left|\bar{\nu}_{y}\right|(R): y \in R\right\} \\
Q(R) & =\sup \left\{\rho^{1-m} \alpha(\rho): 0<\rho<r\right\} \\
K(R) & =\inf \left\{V(R) k^{m-2}+Q(R)\left[\left(\frac{k}{k-1}\right)^{m-1}-1\right]: k>1\right\}
\end{aligned}
$$

Define

$$
\bar{W}_{R}^{*} \mu=\left(\bar{W}^{*} \mu\right) \cap R, \quad \mu \in C^{*}(B) .
$$

Let $C_{R}^{*}$ denote the set of all $\mu \in C^{*}(B)$ enjoying

$$
J(\mu)=\int_{0}^{r} \rho^{1-m}|\mu|\left(R_{\rho}\right) d \rho<\infty
$$

and

$$
|\mu|(B-R)=0
$$

and put

$$
\|\mu\|_{R}=\frac{1}{m-2} r^{2-m}\|\mu\|+J(\mu), \quad \mu \in C_{R}^{*} .
$$

Then $\mu \in C_{R}^{*}$ implies $\bar{W}_{R}^{*} \mu \in C_{R}^{*}$ and

$$
\left\|\bar{W}_{R}^{*} \mu\right\|_{R} \leqq K(R)\|\mu\|_{R} .
$$

Proof. Fix $\mu \in C_{R}^{*}$ and $k>1$. We have with the notation from (4.7)

$$
J(\mu)=\int_{0}^{r} \rho^{1-m} \beta(\rho) d \rho .
$$


Let now $0<\rho<r / k$ and define

$$
\mu_{\rho}=\mu \cap R_{k \rho}, \quad \mu^{\rho}=\mu-\mu_{\rho} .
$$

In view of (4.3)

$$
\left\|\bar{W}_{R}^{*} \mu_{\rho}\right\| \leqq V(R) \beta\left(k_{\rho}\right)
$$

Employing 4.2 we obtain

$$
\left|\bar{W}_{R}^{*} \mu^{\rho}\right|\left(R_{\rho}\right) \leqq \frac{\alpha(\rho) \beta(r)}{(r-\rho)^{m-1}}+(m-1) \alpha(\rho) \int_{k \rho}^{r} \frac{\beta(t) d t}{(t-\rho)^{m}} .
$$

On account of (4.12), (4.13) we get for $0<\rho<r / k$

$$
\rho^{1-m}\left|\bar{W}_{R}^{*} \mu\right|\left(R_{\rho}\right) \leqq V(R) \rho^{1-m} \beta(k \rho)+Q(R) \frac{\beta(r)}{(r-\rho)^{m-1}}+(m-1) Q(R) \int_{k \rho}^{r} \frac{\beta(t) d t}{(t-\rho)^{m}},
$$

while, by (4.3),

$$
\rho^{1-m}\left|\bar{W}_{R}^{*} \mu\right|\left(R_{\rho}\right) \leqq V(R) \rho^{1-m} \beta(r) \quad \text { for } r / k \leqq \rho<r .
$$

Using (4.11) we obtain after simple calculation

$$
\begin{aligned}
J\left(\bar{W}_{R}^{*} \mu\right)= & \int_{0}^{r / k} \rho^{1-m}\left|\bar{W}_{R}^{*} \mu\right|\left(R_{\rho}\right) d \rho+\int_{r / k}^{r} \rho^{1-m}\left|\bar{W}_{R}^{*} \mu\right|\left(R_{\rho}\right) d \rho \\
\leqq & \frac{\beta(r)}{m-2} r^{2-m}\left(V(R)\left(k^{m-2}-1\right)+Q(R)\left[\left(\frac{k}{k-1}\right)^{m-2}-1\right]\right) \\
& +J(\mu)\left(V(R) k^{m-2}+Q(R)\left[\left(\frac{k}{k-1}\right)^{m-1}-1\right]\right) .
\end{aligned}
$$

Since, by virtue of (4.3),

we get finally

$$
\left\|\bar{W}_{R}^{*} \mu\right\| \leqq V(R) \beta(r)
$$

$$
\begin{aligned}
\left\|\bar{W}_{R}^{*} \mu\right\|_{R} & =\frac{1}{m-2} r^{2-m}\left\|\bar{W}_{R}^{*} \mu\right\|+J\left(\bar{W}_{R}^{*} \mu\right) \\
& \leqq\|\mu\|_{R}\left(V(R) k^{m-2}+Q(R)\left[\left(\frac{k}{k-1}\right)^{m-1}-1\right]\right) .
\end{aligned}
$$

\subsection{Notation. Let}

$$
\begin{gathered}
Q_{r}=\sup \left\{\rho^{1-m} H_{m-1}\left(\Omega_{\rho}(z) \cap \hat{B}\right): z \in B, 0<\rho<r\right\}, \quad r>0, \\
Q_{0}=\lim _{r \rightarrow 0+} Q_{r} .
\end{gathered}
$$

Further define $V_{0}$ as in 3.6 and put

$$
K_{0}=\inf \left\{V_{0} k^{m-2}+Q_{0}\left[\left(\frac{k}{k-1}\right)^{m-1}-1\right]: k>1\right\} .
$$


In what follows we shall always assume that

$$
K_{0}<\frac{1}{2} A
$$

4.5. REMARK. The inequality (4.15) implies

$$
V_{0}<\frac{1}{4} A \text {. }
$$

Indeed, since $B$ is infinite and $V_{0}<\frac{1}{2} A$, (3.7) secures $H_{m-1}(\hat{B})>0$. It is known from [DG2], [F3] that for $\left(H_{m-1}\right)$ almost all $y \in \hat{B}$

$$
\lim _{\rho \rightarrow 0+} \rho^{1-m} H_{m-1}\left(\Omega_{\rho}(y) \cap \widehat{B}\right)=b_{m-1},
$$

where $b_{m-1}$ denotes the volume of the unit ball in $R^{m-1}$. Hence $Q_{0} \geqq b_{m-1}$ and $k$ minimizing

$$
V_{0} k^{m-2}+Q_{0}\left[\left(\frac{k}{k-1}\right)^{m-1}-1\right]
$$

must satisfy

$$
\frac{1}{2} A>Q_{0}\left[\left(\frac{k}{k-1}\right)^{m-1}-1\right] \geqq b_{m-1} \frac{m-1}{k-1},
$$

which guarantees $k^{m-2}>2$.

On the other hand, if (4.16) holds, then 3.14 provides an estimate for $Q_{0}$ in terms of $V_{0}$. Clearly, (4.15) is fulfilled whenever $V_{0}$ is sufficiently small.

In view of (4.16) and 3.6, the Fredholm theory applies to the pair of adjoint equations

$$
\begin{aligned}
\left(\frac{1}{2} A I+\bar{W}\right) f & =g, \\
\left(\frac{1}{2} A I+\bar{W}\right)^{*} \mu & =\nu .
\end{aligned}
$$

4.6. LEMMA. If $\mu \in C_{0}^{*}(B)$ then $U|\mu|$ (see the introduction for notation) is bounded on $B$.

Proof. Define $V_{r}$ and $E$ as in 3.6 and fix $r>0$ and $k>1$ such that

$$
\begin{gathered}
K=V_{2 r} k^{m-2}+Q_{r}\left[\left(\frac{k}{k-1}\right)^{m-1}-1\right]<\frac{1}{2} A, \\
r<\operatorname{dist}(E, B-E) .
\end{gathered}
$$

Fix an arbitrary $z \in E$ and define $R=\Omega_{r}(z) \cap B$. We have then with the notation from 4.3

$$
\begin{gathered}
V(R) \leqq V_{2 r}, \quad Q(R) \leqq Q_{r}, \\
K(R) \leqq K<\frac{1}{2} A .
\end{gathered}
$$

Let $\mu \in C_{0}^{*}(B),\|\mu\| \leqq 1$ and put

$$
\mu_{0}=\mu \cap R, \quad \mu^{0}=\mu-\mu_{0} .
$$


In view of (4.2)

$$
\frac{1}{2} A \mu_{0}+\bar{W}^{*} \mu_{0}=-\frac{1}{2} A \mu^{0}-\bar{W}^{*} \mu^{0} .
$$

Restricting all measures occurring in (4.18) to $R$ we obtain

$$
\left(I+2 A^{-1} \bar{W}_{R}^{*}\right) \mu_{0}=-2 A^{-1} \bar{W}_{R}^{*} \mu^{0}
$$

where, of course, $I$ is the identity operator. Employing 4.2 with $\delta=r$ and $\Delta=r$ $+\operatorname{diam} B$ we obtain easily for $0<\rho \leqq r / 2$

$$
\rho^{1-m}\left|\bar{W}^{*} \mu^{0}\right|\left(R_{\rho}\right) \leqq Q_{r} 2^{m} r^{1-m}\left\|\mu^{0}\right\| \leqq Q_{r} 2^{m} r^{1-m} .
$$

On the other hand, we have for $\rho>r / 2$

$$
\rho^{1-m}\left|\bar{W}^{*} \mu^{0}\right|\left(R_{\rho}\right) \leqq 2^{m-1} r^{1-m}\left\|\bar{W}^{*}\right\| \cdot\left\|\mu^{0}\right\| \leqq 2^{m-1} r^{1-m}\left(\frac{1}{2} A+V\right),
$$

so that

$$
J\left(\bar{W}_{R}^{*} \mu^{0}\right) \leqq 2^{m-2} r^{2-m}\left(2 Q_{r}+\frac{1}{2} A+V\right)
$$

Since

$$
\left\|\bar{W}_{R}^{*} \mu^{0}\right\| \leqq\left\|\bar{W}^{*} \mu^{0}\right\| \leqq \frac{1}{2} A+V
$$

we arrive at

$$
\left\|\bar{W}_{R}^{*} \mu^{0}\right\|_{R} \leqq \gamma_{r}
$$

where

$$
\gamma_{r}=\frac{1}{m-2} r^{2-m}\left(\frac{1}{2} A+V\right)+2^{m-2} r^{2-m}\left(2 Q_{r}+\frac{1}{2} A+V\right) .
$$

We see that $\bar{W}_{R}^{*} \mu^{0} \in C_{R}^{*}$. It is easily seen that $C_{R}^{*}$, equipped with the norm $\|\cdots\|_{R}$, is a Banach space. In view of (4.3) and (4.17)

$$
\left\|\bar{W}_{R}^{*}\right\|_{R} \leqq K<\frac{1}{2} A .
$$

Hence we conclude by virtue of (4.19) that $\mu_{0} \in C_{R}^{*}$ and

$$
\left\|\mu_{0}\right\|_{R} \leqq\left(1-\frac{2 K}{A}\right)^{-1} 2 A^{-1} \gamma_{r}=a_{r}
$$

Since $a_{r}$ is independent of $z \in E$, we have, in particular,

$$
\sup _{z \in E} \int_{0}^{r} \rho^{1-m}|\mu|\left(\Omega_{\rho}(z) \cap B\right) d \rho<\infty,
$$

whence it follows easily

$$
\sup _{z \in E} \int_{0}^{\infty} \rho^{1-m}|\mu|\left(\Omega_{\rho}(z) \cap B\right) d \rho<\infty .
$$


Noting that

$$
\begin{aligned}
U|\mu|(z) & =\frac{1}{m-2} \int_{B}|x-z|^{2-m} d|\mu|(x) \\
& =\frac{1}{m-2} \int_{0}^{\infty}|\mu|\left(B \cap\left\{x:|x-z|^{2-m}>t\right\}\right) d t \\
& =\int_{0}^{\infty} \rho^{1-m}|\mu|\left(\Omega_{\rho}(z) \cap B\right) d \rho
\end{aligned}
$$

we see that $U|\mu|$ is bounded on $E$. Since, by 4.1 , spt $\mu \subset E$ and $B-E$ has a positive distance from $E, U|\mu|$ is bounded on $B$ as well.

4.7. Notation. It follows easily from (4.16) and 3.13 that $G$ has only a finite number of components; their closures are mutually disjoint. We shall denote by $q(0 \leqq q<\infty)$ the number of bounded components of $G$. $G_{0}$ will stand for the unbounded component of $G$ (if any); the bounded components of $G$ will be denoted by $G_{1}, \ldots, G_{q}$.

Employing 4.6 we obtain by standard reasoning the following.

\subsection{Lemma. The dimension of $C_{0}^{*}(B)$ does not exceed $q$.}

Proof. Let $\mu \in C_{0}^{*}(B)$. By $4.6, U|\mu|$ is bounded on $B$. Hence it follows that $\mu$ has finite energy [B, p. 122] and

$$
\int_{R^{m}}|\operatorname{grad} U \mu(x)|^{2} d x=A \int_{B} U \mu(y) d \mu(y)<\infty
$$

(see [B, pp. 131, 132]). In particular, there are $\phi_{k} \in D$ such that

$$
\int_{R^{m}}\left|\operatorname{grad} \phi_{k}(x)-\operatorname{grad} U \mu(x)\right|^{2} d x \rightarrow 0 \quad \text { as } k \rightarrow \infty .
$$

(4.2) means that $N U \mu=0$ (see 3.4), so that

$$
\int_{G} \operatorname{grad} \phi_{k}(x) \cdot \operatorname{grad} U \mu(x) d x=0
$$

for each $k$; making $k \rightarrow \infty$ we obtain

$$
\int_{G}|\operatorname{grad} U \mu(x)|^{2} d x=0 .
$$

We see that $U_{\mu}$ is constant on each $G_{j}$ and vanishes on $G_{0}$. Next we prove the following assertion:

(a) If $U_{\mu}=0$ on $G$ then $\mu=0$.

Indeed, let $\mu=\mu_{1}-\mu_{2}$ be the Jordan decomposition of $\mu$ and assume that $U \mu_{1}$ and $U \mu_{2}$ coincide on $G$. Since $G$ has a positive $m$-dimensional density at any $z \in B$, every fine neighborhood of $z$ (in the Cartan topology) meets $G$ (compare 
[B, p. 78, paragraph $2, \S 3$ and p. 84, paragraph 6]) and we conclude from the Cartan Theorem [B, p. 86; see also p. 84] that $U_{\mu_{1}}(z)=U \mu_{2}(z)$. Since $U_{\mu_{1}}$ and $U_{\mu_{2}}$ coincide on $B$, they must coincide on $R^{m}$, by the domination principle [B, p. 123]. We have thus $U_{\mu}=0$ on $R^{m}$, whence $\mu=0$ [B, p. 122].

If $q=0$ then (a) completes the proof of 4.8. Assume now $q>0$. With every $\mu \in C_{0}^{*}(B)$ we may associate the $q$-tuple $c(\mu)=\left[c_{1}(\mu), \ldots, c_{q}(\mu)\right]$, where $c_{f}(\mu)$ is the value taken on by $U \mu$ in $G_{j}$. The map

$$
c: \mu \rightarrow c(\mu)
$$

is an injection of $C_{0}^{*}(B)$ into $R^{q}$. Indeed, $c(\mu)=0$ means that $U_{\mu}=0$ on $G$ and (a) implies $\mu=0$.

4.9. Proposition. Let $f_{j}$ denote the characteristic function of $\operatorname{fr} G_{j}(1 \leqq j \leqq q)$. Then $\left\{f_{1}, \ldots, f_{q}\right\}$ is a basis in $C_{0}(B)$.

Proof. Let us fix $j \in\langle 1, q\rangle$ and put $H=R^{m}-G_{j}$. Employing 2.12 and 2.6 we obtain for any $z \in R^{m}-\operatorname{cl} G \subset$ int $H$

$$
W^{C} f_{j}(z)=\nu_{z}^{H}\left(\mathrm{fr} G_{j}\right)=0,
$$

whence it follows by (3.6)

$$
\left(\frac{1}{2} A I+\bar{W}\right) f_{j}=0,
$$

so that $f_{j} \in C_{0}(B)$. Since the dimension of $C_{0}(B)$ coincides with the dimension of $C_{0}^{*}(B)$ which is known to be $\leqq q$ and $f_{1}, \ldots, f_{q}$ are linearly independent, the proof is complete.

4.10. REMARK. Combining the above proposition and Fredholm's theorems one obtains Theorems 4.11-4.13 below.

If $m=2$ then 4.9 holds under more general assumptions on $B$. It is sufficient to require that $E$ (see 3.6) consists of mutually disjoint simple closed curves and $V_{0}<\frac{1}{2} A$ (compare [K3], where further references may be found).

4.11. TheOREM. Let $\nu \in C^{*}(B)$. Then $\nu=N U \mu$ for some $\mu \in C^{*}(B)$ if and only if

$$
\nu\left(\mathrm{fr} G_{j}\right)=0, \quad j=1, \ldots, q .
$$

Proof. This follows at once from 4.9 and the Fredholm Theorem.

4.12. TheOREM. Let $\left\{f_{1}, \ldots, f_{q}\right\}$ be a basis in $C_{0}(B)$. Given $g \in C(B)$ there are $f \in C(B)$ and constants $\alpha_{j}(j=1, \ldots, q)$ such that, for every $y \in B, W f(x)$ tends to

$$
g(y)-\sum_{j=1}^{q} \alpha_{j} f_{j}(y)
$$

as $x \rightarrow y, x \in$ int $C$. The constants $\alpha_{j}$ are uniquely determined and $f$ is determined modulo $C_{0}(B)$. 
Proof. Let $\left\{\mu_{1}, \ldots, \mu_{q}\right\}$ and $\left\{f_{1}, \ldots, f_{q}\right\}$ be dual bases in $C_{0}^{*}(B)$ and $C_{0}(B)$, respectively. Given $g \in C(B)$ we can find $\alpha_{k}$ so that

$$
\left\langle g-\sum_{k=1}^{q} \alpha_{k} f_{k}, \mu_{j}\right\rangle=0
$$

for all $j$; clearly, $\alpha_{k}=\left\langle g, \mu_{k}\right\rangle$. Then

$$
\left\langle g-\sum_{k=1}^{q} \alpha_{k} f_{k}, C_{0}^{*}(B)\right\rangle=0
$$

and the Fredholm Theorem yields an $f \in C(B)$ such that

$$
\left(\frac{1}{2} A I+\bar{W}\right) f=g-\sum_{k=1}^{q} \alpha_{k} f_{k} .
$$

The rest follows from (3.6).

Standard reasoning yields also the following.

4.13. TheOREm. Fix $x_{j} \in G_{j}(j=1, \ldots, q)$. Given $g \in C(B)$ there are $f \in C(B)$ (determined modulo $C_{0}(B)$ ) and uniquely determined constants $a_{j}$ such that, for every $y \in B$,

$$
W f(x)+\sum_{j=1}^{q} a_{j}\left|x-x_{j}\right|^{2-m}
$$

tends to $g(y)$ as $x \rightarrow y, x \in$ int $C$.

Proof. Define $g_{k}$ by

$$
g_{k}(x)=\frac{1}{m-2}\left|x-x_{k}\right|^{2-m}
$$

Then $\left\langle g_{k}, \mu\right\rangle=U_{\mu}\left(x_{k}\right)$ for every $\mu \in C^{*}(B)$. It follows from (3.6) that

$$
W f+\sum_{j=1}^{q} \alpha_{j} g_{j}\left(f \in C(B), \alpha_{j} \in R^{1}\right)
$$

represents a solution of the Dirichlet problem for $C$ and the boundary condition $g$ if and only if

$$
\left(\frac{1}{2} A I+\bar{W}\right) f=g-\sum_{j=1}^{q} \alpha_{j} g_{j}
$$

on $B$. For the existence of an $f \in C(B)$ satisfying (4.20) it is necessary and sufficient that

$$
\left\langle g-\sum_{j=1}^{q} \alpha_{j} g_{j}, C_{0}^{*}(B)\right\rangle=0,
$$

i.e.,

$$
\sum_{j=1}^{q} \alpha_{j} U \mu\left(x_{j}\right)=\langle g, \mu\rangle, \quad \mu \in C_{0}^{*}(B) .
$$


We know from the proof of 4.8 (note also that $C_{0}^{*}(B)$ has dimension $q$ ) that

$$
\mu \rightarrow\left[U \mu\left(x_{1}\right), \ldots, U \mu\left(x_{q}\right)\right]
$$

is an isomorphism of $C_{0}^{*}(B)$ onto $R^{q}$. Consequently, (4.21) determines $\alpha_{j}$ uniquely. The rest is obvious.

REMARK. Results related to some of those proved in the present paper were announced without proofs in [K1] (for the plane), [BMS] and [MS] (for a domain bounded by a simple closed surface in 3-space), [K2] (for a domain bounded by a hyper-surface in $m$-space) and in Abstract 630-197, (Theorem 1.13), Notices Amer. Math. Soc. 13 (1966).

\section{REFERENCES}

[B] M. Brelot, Éléments de la théorie classique du potentiel, Centre de Documentation Universitaire, Paris, 1961.

[BC] M. Brelot and G. Choquet, Espaces et lignes de Green, Ann. Inst. Fourier (Grenoble) 3 (1952), 199-263.

[BMS] Ju. D. Burago, V. G. Maz'ja and V. D. Sapožnikova, On the potential of a double layer for non-regular domains, Dokl. Akad. Nauk SSSR 147 (1962), 523-525=Soviet Math. Dokl. 3 (1962), 1640-1642.

[CC] C. Constantinescu and A. Cornea, Ideale Ränder Riemannscher Flächen, SpringerVerlag, Berlin, 1963.

[D] J. Deny, Les potentiels d'énergie finie, Acta Math. 82 (1950), 107-183.

[DG1] E. DeGiorgi, Su una teoria generale della misura $(r-1)$-dimensionale in uno spazio ad $r$ dimensioni, Ann. Mat. Pura Appl. (4) 36 (1954), 191-213.

[DG2] - Nuovi teoremi relativi alle misure $(r-1)$-dimensionali in uno spazio ad $r$ dimensioni, Ricerche Mat. 4 (1955), 95-113.

[DG3] - Complementi alla teoria della misura (n-1)-dimensionale in uno spazio ndimensionale, Seminario di Matematica della Scuola Norm. Sup. di Pisa, Anno Accademico, 1960-1961.

[F1] H. Federer, The Gauss-Green theorem, Trans. Amer. Math. Soc. 58 (1945), 44-76.

[F2] - The $(\phi, k)$ rectifiable subsets of $n$-space, Trans. Amer. Math. Soc. 62 (1947), 114-192.

[F3] - A note on the Gauss-Green theorem, Proc. Amer. Math. Soc. 9 (1958), 447-451.

[FF] H. Federer and W. H. Fleming, Normal and integral currents, Ann. of Math. 72 (1960), 458-520.

[FL1] W. H. Fleming, Functions with generalized gradient and generalized surfaces, Ann. Mat. Pura Appl. (4) 54 (1957), 93-104.

[FL2] - Functions whose partial derivatives are measures, Illinois J. Math. 4 (1960), 452-478.

[FY] W. H. Fleming and L. C. Young, Representations of generalized surfaces as mixtures, Rend. Circ. Mat. Palermo (2) 5 (1956), 117-144.

[K1] J. Král, On the logarithmic potential, Comment. Math. Univ. Carolinae 3 (1962), 3-10.

[K2] - On the potential of a double layer in a higher-dimensional space, Dokl. Akad. Nauk SSSR 157 (1964), 1218-1220=Soviet Math. Dokl. 5 (1964), 1677-1680.

[K3] — The Fredholm radius of an operator in potential theory, Czechoslovak Math. J. (90) 15 (1965), 454-473, 565-588. 
[KR] K. Krickeberg, Distributionen, Funktionen beschränkter Variation und Lebesguescher Inhalt nichtparametrischer Flächen, Ann. Mat. Pura. Appl. (4) 44 (1957), 105-134.

[M] F. Y. Maeda, Normal derivatives on an ideal boundary, J. Sci. Hiroshima Univ. Ser. A-1, 28 (1964), 113-131.

[MA] J. Mařík, The surface integral, Czechoslovak Math. J. (81) 6 (1956), 522-558.

[MI] M. Miranda, Distribuzioni aventi derivate misure, Insiemi di perimetro localmente finito, Ann. Scuola Norm. Sup. Pisa (3) 18 (1964), 27-56.

[MS] V. G. Maz'ja and V. D. Sapožnikova, Solving of the Dirichlet and the Neumann problems for non-regular domains by methods of potential theory. Dokl. Akad. Nauk SSSR 159 (1964), 1221-1223 = Soviet Math. Dokl. 5 (1964), 1681-1683.

[P] Chr. Y. Pauc, Functions with generalized gradients in the theory of cell functions, Ann. Mat. Pura. Appl. (4) 44 (1957), 135-152.

[RS] F. Riesz and B. Sz. Nagy, Leçons d'analyse fonctionelle, Akadémiai Kiadó, Budapest, 1952.

[S] L. Schwartz, Théorie des distributions. I, II, Actualités Sci. Ind. Nos. 1091, 1122, Hermann, Paris.

[Y] L. C. Young, A theory of boundary values, Proc. London Math. Soc. (3) 14A (1965), 300-314.

BROWN UNIVERSITY,

Providence, Rhode Island

\section{STATEMENT OF OWNERSHIP,} MANAGEMENT AND CIRCULATION

(Act of October 23, 1962; Section 4369, Title 39, United States Code)

1. Date of filing: September 23, 1966

2. Title of Publication: Transactions of the American Mathematical Society

3. Frequency of issue: Monthly

4. Location of known office of publication: P. O. Box 6248, Providence, R. I. 02904

5. Location of the headquarters or general business offices of the publishers: P. O. Box 6248, Providence, R. I. 02904

6. Names and addresses of publisher, editor and managing editor. Publisher: American Mathematical Society, P. O. Box 6248, Providence, R. I. 02904. Editor: Louis Nirenberg, Chairman of the Editorial Committee, P. O. Box 6248, Providence, R. I. 02904. Managing editor: None

7. Owner: None

8. Known bondholders, mortgagees, and other security holders owning or holding 1 percent or more of total amount of bonds, mortgages or other securities: None

9. Paragraphs 7 and 8 include, in cases where the stockholder or security holder appears upon the books of the company as trustee or in any other fiduciary relation, the name of the person or corporation for whom such trustee is acting, also the statements in the two paragraphs show the affiant's full knowledge and belief as to the circumsts to the circumstances and conditions under which stockholders and security holders who do not appear upon the books of the company as trustees, hold stock and securities in a capacity other than that of a bona fide owner. Names and addresses of individuals who are stockholders of a corporation which itself is a stockholder or holder of bonds, mortgages or other securities of the publishing corporation have been included in paragraphs 7 and 8 when the interests of such individuals are equivalent to 1 percent or more of the total amount of the stock or securities of the publishing corporation.

I certify that the statements made by me above are correct and complete.-Gordon L. Walker 